TRANSACTIONS OF THE

AMERICAN MATHEMATICAL SOCIETY

Volume 364, Number 12, December 2012, Pages 6425-6457

S 0002-9947(2012)05569-3

Article electronically published on June 26, 2012

\title{
THE NON-COMMUTATIVE YOSIDA-HEWITT DECOMPOSITION REVISITED
}

\author{
P. G. DODDS AND B. DE PAGTER
}

\begin{abstract}
In this paper, a new approach to the non-commutative YosidaHewitt decomposition is presented in the general setting of non-commutative symmetric spaces of $\tau$-measurable operators affiliated with semi-finite von Neumann algebras. The principal theorem permits the systematic study of the spaces of normal and singular functionals in this general setting. These results are used to study the properties of elements of order continuous norm and of absolutely continuous norm.
\end{abstract}

\section{INTRODUCTION}

The classical theorem of K. Yosida and E. Hewitt ([29]) asserts that each bounded additive measure can be uniquely represented as the sum of a countably additive measure and a purely finitely additive measure, the so-called singular part. The singular part is characterised by the fact that its absolute value does not dominate any non-zero positive countably additive measure. This classical theorem, which goes back to the Lebesgue decomposition theorem, admits many generalisations in different settings. Within the framework of vector lattices, it emerges as a very special case of the well-known theorem of F. Riesz that a band (that is, an order closed ideal) in an order complete vector lattice is a projection band.

On the other hand, in the study of the Banach dual of a general (non-commutative) von Neumann algebra, it was first shown by M. Takesaki ([24, 25]) that each bounded linear functional $\phi$ on a von Neumann algebra $\mathcal{M}$ could be represented uniquely as the sum of an element $\phi_{n}$ in the predual $\mathcal{M}_{*}$ of $\mathcal{M}$ (which may be identified as the linear space of all completely additive linear functionals on $\mathcal{M}$; see e.g. 17 or 26] ) and an element $\phi_{s}$, which is singular in the sense that it is a linear combination of positive linear functionals, each of which dominates no non-zero positive normal linear functional on $\mathcal{M}$. An important precursor to the paper of Takesaki (and indeed that of Yosida and Hewitt) is the paper of Dixmier [5] who showed that each element of the Banach dual of the space $B(H)$ of all bounded operators on some Hilbert space $H$ admits a unique decomposition into the sum of a normal linear functional and a singular functional which vanishes on the ideal of compact operators.

In the present paper we will show the existence of the Yosida-Hewitt decomposition in the dual space of a symmetric space $E$ of $\tau$-measurable operators affiliated with a semi-finite von Neumann algebra (for precise definitions, see Section (3). Our approach goes well beyond that of [11] and removes the assumption made there that the space $E$ is an interpolation space for the pair $\left(\mathcal{M}, \mathcal{M}_{*}\right)$. Further, the

Received by the editors May 3, 2010 and, in revised form, February 14, 2011.

2010 Mathematics Subject Classification. Primary 46L52, 46L51; Secondary 46E30.

(C)2012 American Mathematical Society 
present approach does not rely on the weak compactness arguments on which the results of [11] are based and, consequently, brings much greater conceptual clarity. In addition, our methods permit a very natural avenue for showing that the space of normal (that is, order continuous) linear functionals $E_{n}^{*}$ on a symmetric space $E$ of $\tau$-measurable operators may be identified with the Köthe dual space $E^{\times}$and that the notions of normality and complete additivity coincide. While this latter equivalence has been established in [10], the present approach is far more transparent.

We give a number of further applications of the study of singular functionals and the Yosida-Hewitt decomposition. In particular, the space of singular functionals $E_{s}^{*}$ is left and right invariant under the action of $\mathcal{M}$ (Corollary [5.10). Furthermore, the spaces $E_{n}^{*}$ and $E_{s}^{*}$ are both sequentially $\sigma\left(E^{*}, E\right)$-complete (Corollary [5.14). Moreover, the inverse annihilator of $E_{s}^{*}$ in $E$ consists precisely of the set $E^{o c}$ of elements of order continuous norm (Proposition 6.2). If $E^{o c}$ separates the points of the Köthe dual $E^{\times}$, then the annihilator of $E^{o c}$ in $E^{*}$ is precisely the space $E_{s}^{*}$ of singular functionals and, in this case, $E^{\times}$may be identified with the Banach dual space of $E^{o c}$ (Proposition 6.4). Furthermore, the space $E^{o c}$ may be identified as the set $E^{a n}$ of all elements in $E$ which are of absolutely continuous norm (Proposition 6.12). An important ingredient in the proof of this result is the fact that a sequence $\left\{x_{n}\right\}_{n=1}^{\infty}$ in $E^{a n}$ converges to zero with respect to the norm of $E$ if and only if $\left\{x_{n}\right\}_{n=1}^{\infty}$ converges to zero with respect to the measure topology and the set $\left\{x_{n}\right\}_{n=1}^{\infty}$ is of uniformly absolutely continuous norm (Theorem 6.11). In the case of (commutative) Banach function spaces, this result goes back to W.A.J. Luxemburg ([20]). We end the paper with a number of characterizations of elements of order continuous norm, which results in corresponding characterizations of symmetric spaces with order continuous norm (Corollary 6.14).

The paper is organized as follows. In Section 2, some terminology is introduced and some necessary background of non-commutative integration theory is provided. In Sections 3 and 4 , (strongly) symmetric spaces of $\tau$-measurable operators are introduced, together with their Köthe duals, and the notions of normal and singular functionals are discussed. The non-commutative Yosida-Hewitt decomposition is proved in Section 5. Finally, in Section 6] some applications of the decomposition are discussed.

Some of the principal results in this paper were announced by the first-named author at a conference on Vector Measures, Integration and Related Topics in Eichstaett, September 2009. Furthermore, an operator version of the Yosida-Hewitt theorem and some related results may be found in [13], to which we refer the interested reader.

\section{Preliminaries}

Let $H$ be a Hilbert space equipped with the inner product $\langle\cdot, \cdot\rangle$. The $C^{*}$-algebra of all bounded linear operators in $H$ is denoted by $B(H)$. The identity operator in $B(H)$ is denoted by $\mathbf{1}$. If $a: \mathfrak{D}(a) \rightarrow H$ is a self-adjoint linear operator, then $e^{a}$ denotes the spectral measure corresponding to $a$. For any Borel function $f: \mathbb{R} \rightarrow \mathbb{C}$, the normal operator $f(a)$ is defined as the spectral integral

$$
f(a)=\int_{\mathbb{R}} f(\lambda) d e^{a}(\lambda)=\int_{\sigma(a)} f(\lambda) d e^{a}(\lambda) .
$$


In particular, for any closed and densely defined linear operator $x: \mathfrak{D}(x) \rightarrow H$ the absolute value is defined by

$$
|x|=\left(x^{*} x\right)^{1 / 2}=\int_{[0, \infty]} \lambda^{1 / 2} d e^{x^{*} x}(\lambda) .
$$

The null projection $n(x)=n(|x|)$ is the (orthogonal) projection onto its kernel Ker $(x)$, the range projection $r(x)$ is the projection onto the closure of its range $\operatorname{Ran}(x)$ and the support projection $s(x)$ of $x$ is defined by $s(x)=\mathbf{1}-n(x)$.

Throughout the present paper, $\mathcal{M} \subseteq B(H)$ will denote a von Neumann algebra on a Hilbert space $H$. For the theory of von Neumann algebras we refer the reader to the books [6, 17, 18, 23, 26] and 27. The unitary group of $\mathcal{M}$ is denoted by $U(M)$, and the complete lattice of all (self-adjoint) projections in $\mathcal{M}$ is denoted by $P(\mathcal{M})$. For any $p \in P(\mathcal{M})$ we write $p^{\perp}=\mathbf{1}-p$. Furthermore, $\mathcal{M}^{\prime}$ is the commutant of $\mathcal{M}$. All von Neumann algebras $\mathcal{M}$ considered in this paper are assumed to be semi-finite, and $\tau$ denotes a fixed semi-finite normal faithful trace on $\mathcal{M}$. The sublattice of $P(\mathcal{M})$ consisting of all $p \in P(\mathcal{M})$ satisfying $\tau(p)<\infty$ is denoted by $P_{f}(\mathcal{M})$.

A linear operator $x: \mathfrak{D}(x) \rightarrow H$, where the domain $\mathfrak{D}(x)$ is a linear subspace of $H$, is called affiliated with $\mathcal{M}$ if $y x \subseteq x y$ for all $y \in \mathcal{M}$ (equivalently, $u x=x u$ for all $u \in U\left(\mathcal{M}^{\prime}\right)$ ); this is denoted by $x \eta \mathcal{M}$. If $x$ is a self-adjoint operator, then $x \eta \mathcal{M}$ if and only if $e^{x}(B) \in P(\mathcal{M})$ for all Borel sets $B \subseteq \mathbb{R}$. If $x$ is a closed and densely defined operator and $x \eta \mathcal{M}$, then the projections $n(x), s(x)$ and $r(x)$ belong to $P(\mathcal{M})$.

A linear subspace $D \subseteq H$ is termed $\tau$-dense if there exists a sequence $\left\{p_{n}\right\}_{n=1}^{\infty} \subseteq$ $P(\mathcal{M})$ such that $p_{n}(H) \subseteq D$ for all $n, p_{n} \uparrow_{n} 1$ and $\tau\left(p_{n}^{\perp}\right)<\infty$ for all $n$. A closed densely defined linear operator $x$ in $H$ is called $\tau$-measurable if $x \eta \mathcal{M}$ and its domain $\mathfrak{D}(x)$ is $\tau$-dense. It should be observed that a closed and densely defined operator $x$ in $H$, which is affiliated with $\mathcal{M}$, is $\tau$-measurable if and only if there exists $\lambda>0$ such that $\tau\left(e^{|x|}(\lambda, \infty)\right)<\infty$. If $x$ is a $\tau$-measurable operator, then the spectral distribution function $d(|x|):[0, \infty) \rightarrow[0, \infty]$ of $|x|$ is defined by $d(s ;|x|)=$ $\tau\left(e^{|x|}(s, \infty)\right), s \geq 0$, and the singular value function $\mu(x):[0, \infty) \rightarrow[0, \infty]$ of $x$ is given by

$$
\mu(t ; x)=\inf \{s \geq 0: d(s ;|x|) \leq t\}, \quad t \geq 0 .
$$

The function $\mu(x)$ is decreasing, right-continuous, and $\mu(t ; x)<\infty$ for all $t>0$. For a discussion of the properties of the singular value function we refer the reader to [14] and [10.

The collection $S(\tau)$ of all $\tau$-measurable operators is a $*$-algebra with respect to strong sum and product (that is, the closure of the algebraic sum and product, respectively). The details may be found in [28. The measure topology $\mathcal{T}_{m}$ in $S(\tau)$ is the vector space topology defined via the neighbourhood base $\{V(\varepsilon, \delta): \varepsilon, \delta>0\}$, where

$$
\begin{aligned}
V(\varepsilon, \delta) & =\left\{x \in S(\tau): \exists p \in P(\mathcal{M}),\|x p\|_{B(H)} \leq \varepsilon, \tau\left(p^{\perp}\right) \leq \delta\right\} \\
& =\left\{x \in S(\tau): \tau\left(e^{|x|}(\varepsilon, \infty)\right) \leq \delta\right\} \\
& =\{x \in S(\tau): \mu(\delta ; x) \leq \varepsilon\} .
\end{aligned}
$$

With respect to $\mathcal{T}_{m}, S(\tau)$ is a complete metrizable topological $*$-algebra (multiplication is jointly continuous, but $\mathcal{T}_{m}$ is not locally convex). It should be observed 
that a net $\left\{x_{\alpha}\right\}$ in $S(\tau)$ converges to zero in measure if and only if $\mu\left(x_{\alpha} ; t\right) \rightarrow_{\alpha} 0$ for all $t>0$. The von Neumann algebra $\mathcal{M}$ is dense in $S(\tau)$ with respect to $\mathcal{T}_{m}$. The extended trace $\tau: S(\tau)^{+} \rightarrow[0, \infty]$ is given by

$$
\tau(x)=\int_{0}^{\infty} \mu(t ; x) d t, \quad x \in S(\tau)^{+}
$$

(see [14 and [10]).

The two-sided ideal $\mathcal{F}(\tau)$ in $\mathcal{M}$ is defined by setting

$$
\mathcal{F}(\tau)=\{x \in \mathcal{M}: \tau(r(x))<\infty\},
$$

and its closure $S_{0}(\tau)$ in $S(\tau)$ with resect to the measure topology is given by

$$
S_{0}(\tau)=\left\{x \in S(\tau): \lim _{t \rightarrow \infty} \mu(t ; x)=0\right\},
$$

which is a two-sided ideal in $S(\tau)$. (Note: in [10] the algebra $S(\tau)$ is denoted by $\widetilde{\mathcal{M}}$; the algebra $\widetilde{\mathcal{M}}_{0}$, as defined in [10], p. 726 , is denoted by $S_{0}(\tau)$ in the present paper.) Furthermore, we note that for every $x \in S(\tau)^{+}$there exists an upwards directed net $\left\{z_{\alpha}\right\}$ in $\mathcal{F}(\tau)^{+}$such that $0 \leq z_{\alpha} \uparrow_{\alpha} x$ (see [10], Proposition 1.8).

The following result turns out to be useful.

Proposition 2.1 ([21], Theorem 5.10). If $\left\{a_{\beta}\right\}$ is an increasing $\mathcal{T}_{m}$-bounded net in $S(\tau)^{+}$, then $\sup _{\beta} a_{\beta} \in S(\tau)^{+}$exists.

The following observations will also be helpful.

Lemma 2.2. If $\left\{a_{\beta}\right\}$ is a downward directed net in $S(\tau)^{+}$such that $a_{\beta} \downarrow_{\beta} 0$, then $x a_{\beta} \stackrel{\mathcal{T}_{m_{\beta}}}{\rightarrow} 0$ and $a_{\beta} x \stackrel{\mathcal{T}}{\rightarrow}_{\beta} 0$ for all $x \in S_{0}(\tau)$.

Proof. Suppose that $a_{\beta_{0}} \geq a_{\beta} \downarrow_{\beta} 0$ in $S(\tau)$ and that $x \in S_{0}(\tau)$. Since $x a_{\beta} x^{*} \downarrow_{\beta} 0$ (see [10, Proposition 1.3) and $0 \leq x a_{\beta} x^{*} \leq x a_{\beta} x^{*} \in S_{0}(\tau)$, it follows from [10, Lemma 3.5, that $x a_{\beta} x^{*} \stackrel{\mathcal{T}}{\rightarrow}_{\beta} 0$. Observing that

$$
\begin{aligned}
\mu\left(x a_{\beta}^{1 / 2}\right)^{2} & =\mu\left(\left|x a_{\beta}^{1 / 2}\right|^{2}\right)=\mu\left(\left(x a_{\beta}^{1 / 2}\right)^{*}\left(x a_{\beta}^{1 / 2}\right)\right) \\
& =\mu\left(\left(x a_{\beta}^{1 / 2}\right)\left(x a_{\beta}^{1 / 2}\right)^{*}\right)=\mu\left(x a_{\beta} x^{*}\right)
\end{aligned}
$$

for all $\beta$, this implies that $x a_{\beta}^{1 / 2} \stackrel{\mathcal{T}_{m_{2}}}{\rightarrow} 0$. Furthermore, $0 \leq a_{\beta}^{1 / 2} \leq a_{\beta_{0}}^{1 / 2}$, which implies that the net $\left\{a_{\beta}^{1 / 2}\right\}$ is bounded with respect to the measure topology. Since $S(\tau)$ is a topological algebra, it follows that $x a_{\beta}=x a_{\beta}^{1 / 2} a_{\beta}^{1 / 2} \stackrel{\mathcal{T}}{\rightarrow}_{\beta} 0$.

Lemma 2.3. If $\left\{x_{n}\right\}_{n=1}^{\infty}$ is a sequence in $S(\tau)$ such that $x_{n} \stackrel{\mathcal{T}_{n}}{\rightarrow} 0$, then there exists a subsequence $\left\{x_{n_{j}}\right\}_{j=1}^{\infty}$ of $\left\{x_{n}\right\}_{n=1}^{\infty}$ and a sequence $\left\{p_{k}\right\}_{k=1}^{\infty}$ in $P(\mathcal{M})$ satisfying $p_{k}(H) \subseteq \mathfrak{D}\left(x_{n_{j}}\right)$ for all $j \geq 1, p_{k} \uparrow_{k} \mathbf{1}$ and $\tau\left(p_{k}^{\perp}\right) \rightarrow 0$ as $k \rightarrow \infty$, such that $\left\|x_{n_{j}} p_{k}\right\|_{B(H)} \rightarrow 0$ as $j \rightarrow \infty$ for all $k$.

Proof. Since $\bigcap_{n=1}^{\infty} \mathfrak{D}\left(x_{n}\right)$ is $\tau$-dense, there exists a sequence $\left\{e_{k}\right\}_{k=1}^{\infty}$ in $P(\mathcal{M})$ such that $e_{k}(H) \subseteq \bigcap_{n=1}^{\infty} \mathfrak{D}\left(x_{n}\right)$ for all $k, e_{k} \uparrow_{k} \mathbf{1}$ and $\tau\left(e_{k}^{\perp}\right) \rightarrow 0$ as $k \rightarrow \infty$. Let $\left\{x_{n_{j}}\right\}_{j=1}^{\infty}$ be a subsequence of $\left\{x_{n}\right\}_{n=1}^{\infty}$ satisfying $x_{n_{j}} \in V\left(2^{-j}, 2^{-j}\right)$ for all $j \geq 1$. For each $j$ there exists $q_{j} \in P(\mathcal{M})$ such that $\left\|x_{n_{j}} q_{j}\right\|_{B(H)} \leq 2^{-j}$ and $\tau\left(q_{j}^{\perp}\right) \leq 2^{-j}$. 
Defining $p_{k}=\left(\bigwedge_{j=k}^{\infty} q_{j}\right) \wedge e_{k}$ for $k \geq 1$, it is easily verified that $\left\{x_{n_{j}}\right\}_{j=1}^{\infty}$ and $\left\{p_{k}\right\}_{k=1}^{\infty}$ satisfy the conclusion of the lemma.

Another important vector space topology on $S(\tau)$ is the local measure topology $\mathcal{T}_{l m}$. A neighbourhood base for this topology is given by the sets $V(\varepsilon, \delta ; e), \varepsilon, \delta>0$, $e \in P_{f}(\mathcal{M})$, where

$$
V(\varepsilon, \delta ; e)=\{x \in S(\tau): \text { exe } \in V(\varepsilon, \delta)\} .
$$

Since $V(\varepsilon, \delta) \subseteq V(\varepsilon, \delta ; e)$, it is clear that $\mathcal{T}_{l m}$ is weaker than $\mathcal{T}_{m}$. Evidently, a net $\left\{x_{\alpha}\right\}$ in $S(\tau)$ converges locally in measure (that is, with respect to $\mathcal{T}_{l m}$ ) to $x \in S(\tau)$ if and only if $e x_{\alpha} e \stackrel{\mathcal{T}_{m_{2}}}{\rightarrow_{\alpha}}$ exe for all $e \in P_{f}(\mathcal{M})$. In general, $\mathcal{T}_{l m}$ is not metrizable and multiplication in $S(\tau)$ is not jointly continuous with respect to $\mathcal{T}_{l m}$. However, if $\left\{x_{\alpha}\right\} \subseteq S(\tau)$ is a net and if $x_{\alpha} \stackrel{\mathcal{T}_{l m}}{\rightarrow} x \in S(\tau)$, then $y x_{\alpha}{\stackrel{\mathcal{T}_{l m}}{\rightarrow}}_{\alpha} y x$ and $x_{\alpha} y{\stackrel{\mathcal{T}_{l m}}{\rightarrow}}_{\alpha} x y$ for all $y \in S(\tau)$ (see [2]). Furthermore, it should be observed that if $0 \leq x_{\alpha} \uparrow_{\alpha} x$ in $S(\tau)$ (that is, $\left\{x_{\alpha}\right\}$ is an increasing net in $S(\tau)^{+}$and $\sup _{\alpha} x_{\alpha}=x \in S(\tau)^{+}$), then $x_{\alpha} \stackrel{\mathcal{T}_{l m}}{\rightarrow} x$. Indeed, if $e \in P_{f}(\mathcal{M})$, then $0 \leq e x_{\alpha} e \uparrow_{\alpha}$ exe and exe $\in S_{0}(\tau)^{+}$. Therefore, it follows from [10], Lemma 3.5, that $e x_{\alpha} e \stackrel{\mathcal{T}_{m}}{\rightarrow}$ exe.

It will be convenient to have a Fatou-type lemma available for the local measure topology. If $\left\{t_{\alpha}\right\}$ is any net in $\mathbb{R}$, then we define $\liminf \inf _{\alpha} t_{\alpha}=\sup _{\beta}\left(\inf _{\alpha \geq \beta} t_{\alpha}\right)$ (which always exists in $[-\infty, \infty]$ ).

Lemma 2.4. If $\left\{x_{\alpha}\right\}$ is a net in $S(\tau)$ and if $x \in S(\tau)$ is such that $x_{\alpha} \stackrel{\mathcal{T}_{l m}}{\rightarrow} x$, then

$$
\tau(|x|) \leq \liminf \operatorname{in}_{\alpha}\left(\left|x_{\alpha}\right|\right) .
$$

Proof. First, we prove (2.3) under the stronger assumption that $x_{\alpha} \stackrel{\mathcal{T}_{m}}{{ }_{\alpha}} x$. Without loss of generality, it may be assumed that $\liminf _{\alpha} \tau\left(\left|x_{\alpha}\right|\right)=C<\infty$. From the definition of convergence in measure (that is, convergence with respect to $\mathcal{T}_{m}$ ) it follows that there exists an increasing sequence of indices $\left\{\beta_{n}\right\}_{n=1}^{\infty}$ such that $x-x_{\alpha} \in$ $V\left(\frac{1}{n}, \frac{1}{n}\right)$ whenever $\alpha \geq \beta_{n}$. Given $\varepsilon>0$, for each $n$ we have $\inf _{\alpha \geq \beta_{n}} \tau\left(\left|x_{\alpha}\right|\right)<C+\varepsilon$, and so there exists $\alpha_{n} \geq \beta_{n}$ such that $\tau\left(\left|x_{\alpha_{n}}\right|\right)<C+\varepsilon$. It is clear that $x_{\alpha_{n}} \stackrel{\mathcal{T}_{n}}{\rightarrow} x$ as $n \rightarrow \infty$, and hence it follows from Fatou's lemma for sequences (see 14, Theorem $3.5)$ that $\tau(|x|) \leq \liminf _{n \rightarrow \infty} \tau\left(\left|x_{\alpha_{n}}\right|\right) \leq C+\varepsilon$. Since this holds for all $\varepsilon>0$, we may conclude that $\tau(|x|) \leq C$.

Suppose now that $x_{\alpha} \stackrel{\overline{\mathcal{T}}_{\text {lm }}}{\rightarrow} \alpha$. If $x=v|x|$ is the polar decomposition of $x$, then it follows that $v^{*} x_{\alpha} \stackrel{\mathcal{T}_{l m}}{\rightarrow} v^{*} x=|x|$, and therefore, if $e \in P_{f}(\mathcal{M})$, then $e v^{*} x_{\alpha} e \stackrel{\mathcal{T}_{m}}{\rightarrow}{ }_{\alpha} e|x| e$. By the first part of the proof, this implies that

$$
\tau(e|x| e) \leq \liminf _{\alpha} \tau\left(\left|e v^{*} x_{\alpha} e\right|\right) \leq \liminf _{\alpha} \tau\left(\left|x_{\alpha}\right|\right) .
$$

It follows easily from [10], Lemma 1.5 (observing that the supremum in this lemma is taken over an upwards directed family) that

$$
\tau(|x|)=\sup \left\{\tau(e|x| e): e \in P_{f}(\mathcal{M})\right\},
$$

and so we may conclude that $\tau(|x|) \leq \liminf _{\alpha} \tau\left(\left|x_{\alpha}\right|\right)$. The proof is complete.

We recall the following notion of submajorization in the sense of Hardy, Littlewood and Polya. Suppose that $\mathcal{M}_{1}$ and $\mathcal{M}_{2}$ are von Neumann algebras on Hilbert spaces $H_{1}$ and $H_{2}$, respectively, equipped with semi-finite normal faithful traces $\tau_{1}$ 
and $\tau_{2}$, respectively. If $x \in S\left(\tau_{1}\right)$ and $y \in S\left(\tau_{2}\right)$, then $x$ is said to be submajorized by $y$, denoted by $x \prec \prec y$, if

$$
\int_{0}^{t} \mu(s ; x) d s \leq \int_{0}^{t} \mu(s ; y) d s
$$

for all $t \geq 0$.

The following result plays an important role in the study of Köthe duality. It is a slight extension of [10, Theorem 4.12 (i), from which it can be easily obtained.

Proposition 2.5. If $x, y \in S(\tau)$, then

$$
\int_{0}^{\infty} \mu(t ; x) \mu(t ; y) d t=\sup \{\tau(|z y|): z \in \mathcal{F}(\tau), z \prec \prec y\} .
$$

\section{Symmetric sPaCes AND their Köthe DUals}

As before, $\mathcal{M}$ is a semi-finite von Neumann algebra on a Hilbert space $H$, equipped with a fixed semi-finite normal faithful trace $\tau$.

Definition 3.1. $\quad$ (i) A linear subspace $E$ of $S(\tau)$, equipped with a norm $\|\cdot\|_{E}$, is called symmetrically normed if $x \in S(\tau), y \in E$ and $\mu(x) \leq \mu(y)$ imply that $x \in E$ and $\|x\|_{E} \leq\|y\|_{E}$. If, in addition, $E$ is a Banach space, then $E$ is termed a symmetric space (of $\tau$-measurable operators).

(ii) A symmetrically normed space $E \subseteq S(\tau)$ is called strongly symmetrically normed if its norm $\|\cdot\|_{E}$ has the additional property that $\|x\|_{E} \leq\|y\|_{E}$ whenever $x, y \in E$ satisfy $x \prec \prec y$. Moreover, if $E$ is also a Banach space, then $E$ is called a strongly symmetric space.

(iii) A linear subspace $E$ of $S(\tau)$, equipped with a norm $\|\cdot\|_{E}$, is called fully symmetrically normed if $x \in S(\tau), y \in E$ and $x \prec \prec y$ imply that $x \in E$ and $\|x\|_{E} \leq\|y\|_{E}$. If, in addition, $E$ is a Banach space, then $E$ is called a fully symmetric space.

Evidently, any fully symmetrically normed space is strongly symmetrically normed.

Remark 3.2. In the present paper, for the sake of simplicity, we shall only consider strongly symmetrically normed spaces. Actually, all results are also valid for symmetrically normed spaces under the additional assumption that the von Neumann algebra $\mathcal{M}$ either does not contain any atoms (that is, minimal projections) or $\mathcal{M}$ is discrete and all minimal projections have equal trace.

Examples of fully symmetric spaces are the non-commutative $L_{p}$-spaces $L_{p}(\tau)$, $1 \leq p \leq \infty$ (where $\left.L_{\infty}(\tau)=\mathcal{M}\right), L_{1}(\tau) \cap L_{\infty}(\tau)$ and $L_{1}(\tau)+L_{\infty}(\tau)$ (equipped with their usual norms). Note that $\mathcal{F}(\tau) \subseteq L_{1}(\tau) \cap L_{\infty}(\tau)$ and that $\mathcal{F}(\tau)$ is fully symmetrically normed, where the norm $\|\cdot\|_{\mathcal{F}(\tau)}$ is given by $\|x\|_{\mathcal{F}(\tau)}=\|x\|_{L_{1} \cap L_{\infty}}$, $x \in \mathcal{F}(\tau)$.

Given a strongly symmetrically normed space $E \subseteq S(\tau)$, let $P(E)=P(\mathcal{M}) \cap E$, which is a solid sublattice of $P(\mathcal{M})$. The projection $c_{E}=\bigvee P(E)$ is called the carrier projection of $E$. It is easily verified that $c_{E}$ is a central projection. To avoid trivialities, it will always be assumed that $c_{E}=\mathbf{1}$. Under this condition, it may be shown that

$$
\mathcal{F}(\tau) \subseteq E \subseteq L_{1}(\tau)+L_{\infty}(\tau)
$$


with continuous embeddings. If $E$ is a strongly symmetric space, then the continuous embedding $L_{1}(\tau) \cap L_{\infty}(\tau) \subseteq E$ is always valid. We will omit the proofs of these assertions, as they are somewhat technical. However, for spaces $E$ constructed from strongly symmetric spaces on the half-line $(0, \infty)$ (see below), these embeddings follow directly from the corresponding embeddings for symmetric spaces on $(0, \infty)$. See for example [19], Theorem II.4.1.

Remark 3.3. We would like to warn the reader that the terminology introduced above differs from that which has been used elsewhere and is an attempt to unify some of the terminology in the literature. We point out explicitly that the terms "symmetrically normed" and "strongly symmetrically normed" as defined above are used in the present paper instead of the earlier terminology of "rearrangement invariant" and "symmetrically normed", respectively, used in 9. and [10. In particular, strongly symmetric spaces $E$ satisfying $c_{E}=\mathbf{1}$ (as we shall always assume) correspond to properly symmetric spaces, as introduced in [10, Section 5.

An important subspace of a strongly symmetrically normed space $E$ is given by $E^{b}=\overline{\mathcal{F}}(\tau)^{E}$, the norm closure of $\mathcal{F}(\tau)$ in $E$. If $E$ is complete, then

$$
E^{b}={\overline{L_{1}(\tau) \cap L_{\infty}(\tau)}}^{E} .
$$

Furthermore, it is clear that $u x v \in E^{b}$ whenever $x \in E$ and $u, v \in \mathcal{M}$. This implies, in particular, that if $x \in S(\tau)$, then $x \in E^{b}$ if and only if $|x| \in E^{b}$.

Proposition 3.4. For $x \in E$ the following statements are equivalent:

(i) $x \in E^{b}$;

(ii) $x \in S_{0}(\tau),\left\|x e^{|x|}(0, s]\right\|_{E} \rightarrow 0$ as $s \downarrow 0$, and $\left\|x e^{|x|}(s, \infty)\right\|_{E} \rightarrow 0$ as $s \rightarrow \infty$.

Furthermore, if $x \in S(\tau)$ and $y \in E^{b}$ are such that $\mu(x) \leq \mu(y)$, then $y \in E^{b}$ (consequently, $\left(E^{b},\|\cdot\|_{E}\right)$ is a strongly symmetrically normed space in its own right).

Proof. If $x \in E$ satisfies (ii) and we define $z_{n}=x e^{|x|}(1 / n, n]$, then it is easily verified that the sequence $\left\{z_{n}\right\}_{n=1}^{\infty}$ belongs to $\mathcal{F}(\tau)$ and that $\left\|x-z_{n}\right\|_{E} \rightarrow 0$ as $n \rightarrow \infty$. Hence, $x \in E^{b}$.

Since $\mathcal{F}(\tau) \subseteq S_{0}(\tau)$ and the embedding of $E$ into $S(\tau)$ is continuous with respect to the measure topology, it is clear that $E^{b} \subseteq S_{0}(\tau)$. Suppose now that $x \in E^{b}$. To show that $x$ satisfies the two remaining statements of (ii), we may assume, without loss of generality, that $x \geq 0$. Given $\varepsilon>0$, let $y \in \mathcal{F}(\tau)$ be such that $\|x-y\|_{E} \leq \varepsilon$. Replacing $y$ by $\operatorname{Re} y$, we may assume that $y^{*}=y$. Since $0 \leq y^{-} \leq e^{y}(0, \infty)(x-y) e^{y}(0, \infty)$, it follows that $\left\|y^{-}\right\|_{E} \leq \varepsilon$, and so $\left\|x-y^{+}\right\|_{E} \leq 2 \varepsilon$. Therefore, we may assume that $y \in \mathcal{F}(\tau)^{+}$. With $p=r(y)$ we have $y=p y$ and $\tau(p)<\infty$. Since $\left\|x p^{\perp}\right\|_{E} \leq \varepsilon$, it follows that

$$
\left\|x e^{|x|}(0, s]\right\|_{E} \leq \varepsilon+\left\|p x e^{|x|}(0, s]\right\|_{E} \leq \varepsilon+s\|p\|_{E}, \quad s>0,
$$

from which it may be concluded that $\left\|x e^{|x|}(0, s]\right\|_{E} \rightarrow 0$ as $s \downarrow 0$. Suppose now that $s>2\|y\|_{\infty}$, and, for convenience, put $e=e^{x}(s, \infty)$. Defining $z=$ eye, it is clear that $\|x e-z\|_{E} \leq \varepsilon$ and $0 \leq z \leq\|y\|_{\infty} e \leq s e \leq x e$. Hence, $0 \leq$ $\left(s-\|y\|_{\infty}\right) e \leq x e-z$, and so $\|e\|_{E} \leq\left(s-\|y\|_{E}\right)^{-1} \varepsilon$, which implies that $\|z\|_{E}=$ $\|z e\|_{E} \leq\|z\|_{\infty}\|e\|_{E} \leq \varepsilon$. Consequently, $\|x e\|_{E} \leq\|x e-z\|_{E}+\|z\|_{E} \leq 2 \varepsilon$, and we may conclude that $\left\|x e^{|x|}(s, \infty)\right\|_{E} \rightarrow 0$ as $s \rightarrow \infty$. This shows that statements (i) and (ii) are equivalent. 
Suppose now that $x \in S(\tau)$ and $y \in E^{b}$ are such that $\mu(x) \leq \mu(y)$. By the above, $y$ satisfies the conditions of (ii), and it suffices to show that $x$ also satisfies (ii). It is clear that $x \in S_{0}(\tau)$. Given $s>0$, set $\alpha=d(s ;|x|)$ and $\beta=d(s ;|y|)$ and note that $\alpha \leq \beta$. Consequently,

$$
\mu\left(|x| e^{|x|}(s, \infty)\right)=\mu(x) \chi_{[0, \alpha)} \leq \mu(y) \chi_{[0, \beta)}=\mu\left(|y| e^{|y|}(s, \infty)\right),
$$

and so $\left\||x| e^{|x|}(s, \infty)\right\|_{E} \leq\left\||y| e^{|y|}(s, \infty)\right\|_{E}$. This implies that $\left\|x e^{|x|}(s, \infty)\right\|_{E} \rightarrow 0$ as $s \rightarrow \infty$. If $\mu(t ; x)=0$ for some $t>0$, then $\left\|x e^{|x|}(0, s]\right\|_{E} \rightarrow 0$ as $s \downarrow 0$. Indeed, in this case $\tau\left(e^{|x|}(0, \infty)\right) \leq t$, and so $e^{|x|}(0, \infty) \in E$, which implies that $\left\|x e^{|x|}(0, s]\right\|_{E} \leq s\left\|e^{|x|}(0, \infty)\right\|_{E}$. This proves our claim. Therefore, to show that $x$ satisfies the second condition of (ii), it may be assumed that $\mu(t ; x)>0$ for all $t>0$ (and hence, $\mu(t ; y)>0, t>0)$. If $0<s_{1}<\mu(\beta ; y)$, then $\alpha_{1}=d\left(s_{1} ;|x|\right)$ satisfies $\alpha_{1} \geq \beta$, and hence

$$
\mu\left(t ;|x| e^{|x|}\left(0, s_{1}\right]\right)=\mu\left(t+\alpha_{1} ; x\right) \leq \mu(t+\beta ; y)=\mu\left(t ;|y| e^{|y|}(0, s]\right), \quad t>0 .
$$

This implies that $\left\|x e^{|x|}\left(0, s_{1}\right]\right\|_{E} \leq\left\|y e^{|y|}(0, s]\right\|_{E}$ whenever $0<s_{1}<\mu(\beta ; y)$, where $\beta=d(s ;|y|)$. Therefore, we may conclude that $\left\|x e^{|x|}(0, s]\right\|_{E} \rightarrow 0$ as $s \downarrow 0$. The proof is complete.

Remark 3.5. As follows from the above proposition, if $E$ is a strongly symmetrically normed space, then $E^{b}$ is also a strongly symmetrically normed space. It may be shown that if $E$ is a strongly symmetric space, then $E^{b}$ is a fully symmetric space (that is, $x \in S(\tau), y \in E^{b}$ and $x \prec \prec y$ imply that $x \in E^{b}$ ). The proof of this fact is much more complicated.

If $E \subseteq S(\tau)$ is a strongly symmetrically normed space, then the Köthe dual $E^{\times} \subseteq S(\tau)$ is defined by setting

$$
E^{\times}=\left\{y \in S(\tau):\|y\|_{E^{\times}}<\infty\right\},
$$

where

$$
\|y\|_{E^{\times}}=\sup \left\{\tau(|x y|): x \in E,\|x\|_{E} \leq 1\right\} .
$$

Remark 3.6. $\quad$ (i) It should be observed that

$$
\|y\|_{E^{\times}}=\sup \left\{\tau(|x y|): x \in \mathcal{F}(\tau),\|x\|_{E} \leq 1\right\}, \quad y \in S(\tau) .
$$

Indeed, fix $y \in S(\tau)$ and let $A$ denote the supremum on the right hand side. It is clear that $A \leq\|y\|_{E^{\times}}$. Given $x \in E$ with $\|x\|_{E} \leq 1$ and polar decomposition $x=v|x|$, there exists a net $\left\{z_{\alpha}\right\}$ in $\mathcal{F}(\tau)^{+}$such that $0 \leq z_{\alpha} \uparrow_{\alpha}|x|$. As observed above, this implies that $v z_{\alpha} y \stackrel{\mathcal{T}}{\longrightarrow m}_{\alpha} x y$. For each $\alpha$ we have $v z_{\alpha} \in \mathcal{F}(\tau)$ and $\left\|v z_{\alpha}\right\|_{E} \leq 1$, hence $\tau\left(\left|v z_{\alpha} y\right|\right) \leq A$. By Lemma 2.4 it follows that $\tau(|x y|) \leq A$. This shows that $\|y\|_{E^{\times}} \leq A$, and the claim is proved.

(ii) If $E, F \subseteq S(\tau)$ are strongly symmetrically normed spaces such that $F \subseteq E$, with continuous embedding, then it is easily verified that $F^{\times} \subseteq E^{\times}$, with continuous embedding. In particular, since $\mathcal{F}(\tau) \subseteq E \subseteq L_{1}(\tau)+L_{\infty}(\tau)$, with continuous embeddings, it follows that

$$
\left(L_{1}(\tau)+L_{\infty}(\tau)\right)^{\times} \subseteq E^{\times} \subseteq \mathcal{F}(\tau)^{\times},
$$


with continuous embeddings. It follows, by direct computation, that

$$
\left(L_{1}(\tau)+L_{\infty}(\tau)\right)^{\times}=L_{1}(\tau) \cap L_{\infty}(\tau) \quad \text { and } \quad \mathcal{F}(\tau)^{\times}=L_{1}(\tau)+L_{\infty}(\tau)
$$

as Banach spaces. Consequently,

$$
L_{1}(\tau) \cap L_{\infty}(\tau) \subseteq E^{\times} \subseteq L_{1}(\tau)+L_{\infty}(\tau) .
$$

It follows from Proposition 2.5 and Remark 3.6 (i) that

$$
\|y\|_{E^{\times}}=\sup \left\{\int_{0}^{\infty} \mu(t ; x) \mu(t ; y) d t: x \in E,\|x\|_{E} \leq 1\right\}, \quad y \in S(\tau) .
$$

If $E \subseteq S(\tau)$ is a strongly symmetrically normed space, then $E$ is said to have the Fatou property if and only if whenever $\left\{x_{\alpha}\right\} \subseteq E^{+}$is an upwards directed system for which $\sup _{\alpha}\left\|x_{\alpha}\right\|_{E}<\infty$, it follows that there exists $x \in E^{+}$such that $0 \leq x_{\alpha} \uparrow_{\alpha} x$ and $\|x\|_{E}=\sup _{\alpha}\left\|x_{\alpha}\right\|_{E}$. We will now show that the Köthe dual of a strongly symmetrically normed space always has the Fatou property. This result was first established in [10, Proposition 5.4 and Corollary 5.12. However, the proof in [10] is somewhat indirect, as it depends on the identification of the Köthe dual $E^{\times}$with the space of all order continuous (that is, normal) functionals on $E$. It is, therefore, preferable to indicate a simplified proof based on Proposition 2.1.

Proposition 3.7. If is $E \subseteq S(\tau)$ a strongly symmetrically normed space, then $E^{\times}$ is a fully symmetric space with the Fatou property.

Proof. If $y \in E^{\times}$and $z \in S(\tau)$ are such that $z \prec \prec y$, then it follows from a well known inequality (due to Hardy) that

$$
\int_{0}^{\infty} \mu(t ; x) \mu(t ; z) d t \leq \int_{0}^{\infty} \mu(t ; x) \mu(t ; y) d t, \quad x \in E,\|x\|_{E} \leq 1
$$

and hence, (3.1) implies that $z \in E^{\times}$and $\|z\|_{E^{\times}} \leq\|y\|_{E^{\times}}$.

Suppose that $\left\{y_{\alpha}\right\}$ is an upwards directed system in $E^{+}$such that $\sup _{\alpha}\left\|y_{\alpha}\right\|_{E^{\times}}$ $<\infty$. Since the embedding of $E^{\times}$into $S(\tau)$ is continuous (with respect to the norm topology in $E^{\times}$and the measure topology in $S(\tau)$ ), it is clear that $\left\{y_{\alpha}\right\}$ is $\mathcal{T}_{m}$-bounded. Therefore, it follows from Proposition 2.1 that there exists $y \in S(\tau)^{+}$ such that $0 \leq y_{\alpha} \uparrow_{\alpha} y$. This implies that $\mu\left(y_{\alpha}\right) \uparrow_{\alpha} \mu(y)$ in $L_{0}(0, \infty)$, and so

$$
\begin{aligned}
\|y\|_{E^{\times}} & =\sup _{x \in E,\|x\|_{E} \leq 1} \int_{0}^{\infty} \mu(t ; x) \mu(t ; y) d t \\
& =\sup _{x \in E,\|x\|_{E} \leq 1} \sup _{\alpha} \int_{0}^{\infty} \mu(t ; x) \mu\left(t ; y_{\alpha}\right) d t \\
& =\sup _{\alpha} \sup _{x \in E,\|x\|_{E} \leq 1} \int_{0}^{\infty} \mu(t ; x) \mu\left(t ; y_{\alpha}\right) d t=\sup _{\alpha}\left\|y_{\alpha}\right\|_{E^{\times}} .
\end{aligned}
$$

Hence, $E^{\times}$has the Fatou property. The Fatou property implies that $E^{\times}$is a Banach space (see [10], Corollary 2.4), and so $E^{\times}$is a fully symmetric space. The proof is complete.

Let $m$ denote Lebesgue measure on $(0, \infty)$ and suppose that $E(0, \infty) \subseteq S(m)$ is a strongly symmetric space. If $(\mathcal{M}, \tau)$ is a semi-finite von Neumann algebra and we define

$$
E(\tau)=\{x \in S(\tau): \mu(x) \in E\}
$$


and

$$
\|x\|_{E(\tau)}=\|\mu(x)\|_{E(0, \infty)}, \quad x \in E(\tau),
$$

then $\left(E(\tau),\|\cdot\|_{E(\tau)}\right)$ is a strongly symmetric space (see [7], 8]). Furthermore,

$$
E(\tau)^{\times}=E^{\times}(\tau),
$$

where $E^{\times}=E(0, \infty)^{\times}$(see [10], Theorem 5.6). This implies, in particular, that $L_{p}(\tau)^{\times}=L_{q}(\tau)$, where $1 \leq p \leq \infty$ and $1 / p+1 / q=1$. Similarly, $\left(L_{1}+L_{\infty}\right)(\tau)^{\times}=$ $\left(L_{1} \cap L_{\infty}\right)(\tau)$ and $\left(L_{1} \cap L_{\infty}\right)(\tau)^{\times}=\left(L_{1}+L_{\infty}\right)(\tau)$, where it should be observed that $\left(L_{1}+L_{\infty}\right)(\tau)=L_{1}(\tau)+L_{\infty}(\tau)$ and $\left(L_{1} \cap L_{\infty}\right)(\tau)=L_{1}(\tau) \cap L_{\infty}(\tau)$, as Banach spaces.

\section{NORMAL AND SINGULAR FUNCTIONALS}

As before, we assume that $(\mathcal{M}, \tau)$ is a semi-finite von Neumann algebra and that $E \subseteq S(\tau)$ is a strongly symmetrically normed space (satisfying $c_{E}=\mathbf{1}$ ). The Banach space dual of $E$ is denoted by $E^{*}$. If $\phi \in E^{*}$ and $w \in \mathcal{M}$, then the functionals $\phi w, w \phi \in E^{*}$ are defined by $(\phi w)(x)=\phi(w x)$ and $(w \phi)(x)=\phi(x w)$, $x \in E$, respectively. For any $\phi \in E^{*}$, the conjugate functional $\bar{\phi} \in E^{*}$ is defined by $\bar{\phi}(x)=\overline{\phi\left(x^{*}\right)}, x \in E$. The functional $\phi$ is called hermitian if $\bar{\phi}=\phi$, and the set of all hermitian functionals is denoted by $E_{h}^{*}$, which is a real linear subspace of $E^{*}$. For $\phi \in E^{*}$ we define the hermitian functionals $\operatorname{Re} \phi$ and $\operatorname{Im} \phi$ by setting

$$
\operatorname{Re} \phi=\frac{1}{2}(\phi+\bar{\phi}) \quad \text { and } \operatorname{Im} \phi=\frac{1}{2 i}(\phi-\bar{\phi}),
$$

in which case $\phi=\operatorname{Re} \phi+i \operatorname{Im} \phi$. Hence, $E^{*}=E_{h}^{*} \oplus i E_{h}^{*}$. As usual, a functional $\phi \in E^{*}$ is called positive if $\phi(x) \geq 0$ whenever $x \in E^{+}$. Note that every positive functional is hermitian. The proper closed cone of all positive functionals is denoted by $\left(E^{*}\right)^{+}$, and with respect to this cone, $E_{h}^{*}$ is an ordered Banach space. Note that $E_{h}^{*}$ may be identified (isometrically) with the dual of the (real) ordered normed space $E_{h}$.

It should be observed that $E^{+}$is a 2-normal cone; that is, it follows from $a \leq b \leq$ $c$ in $E_{h}$ that $\|b\|_{E} \leq 2 \max \left(\|a\|_{E},\|c\|_{E}\right)$. Indeed, it follows from $b \leq c$ that $0 \leq b^{+}=$ $e^{b}[0, \infty) b e^{b}[0, \infty) \leq e^{b}[0, \infty) c e^{b}[0, \infty)$, and so $\left\|b^{+}\right\|_{E} \leq\left\|e^{b}[0, \infty) c e^{b}[0, \infty)\right\|_{E} \leq$ $\|c\|_{E}$. Similarly, $-b \leq-a$ implies that $\left\|b^{-}\right\|_{E} \leq\|a\|_{E}$, from which the claim follows. Consequently, it follows from a theorem of J. Grosberg and M. Krein ([15]; see [16], Theorem 3.6.7; also [1]) that the positive cone $\left(E^{*}\right)^{+}$is 2-generating in $E_{h}^{*}$; that is, every $\phi \in E_{h}^{*}$ has a decomposition

$$
\phi=\phi_{1}-\phi_{2}, \quad \phi_{1}, \phi_{2} \in\left(E^{*}\right)^{+},\left\|\phi_{1}\right\|_{E^{*}}+\left\|\phi_{2}\right\|_{E^{*}} \leq 2\|\phi\|_{E^{*}} .
$$

The following result is a simple consequence.

Corollary 4.1. If $x \in E$, then

$$
\|x\|_{E} \leq 4 \sup \left\{|\phi(x)|: \phi \in\left(E^{*}\right)^{+},\|\phi\|_{E^{*}} \leq 1\right\} .
$$

The following inequality will prove to be useful in the sequel.

Lemma 4.2. If $\phi \in\left(E^{*}\right)^{+}$, then

$$
|\phi(w x)|^{2} \leq \phi\left(w\left|x^{*}\right| w^{*}\right) \phi(|x|), \quad x \in E, w \in \mathcal{M} .
$$


Proof. Define the subset $F \subseteq S(\tau)$ by

$$
F=\left\{x \in S(\tau):|x|^{2} \in E\right\} .
$$

By the same method of proof as used in [6] I.1.6, it may be shown that $F$ is a linear subspace of $S(\tau)$ with the property that $v x w \in F$ whenever $x \in F$ and $v, w \in \mathcal{M}$ (which implies, in particular, that $F$ is $*$-closed), satisfying

$$
E=F^{2}=\left\{\sum_{j=1}^{n} x_{j} y_{j}: x_{j}, y_{j} \in F, 1 \leq j \leq n ; n \in \mathbb{N}\right\} .
$$

If $x, y \in F$, then $y^{*} x \in E$, and so we may define the semi-inner product $\langle\cdot, \cdot\rangle_{\phi}$ in $F$ by $\langle x, y\rangle_{\phi}=\phi\left(y^{*} x\right), x, y \in F$. Hence, by Cauchy-Schwarz,

$$
\left|\phi\left(y^{*} x\right)\right|^{2} \leq \phi\left(x^{*} x\right) \phi\left(y^{*} y\right), \quad x, y \in F .
$$

Now, let $x \in E$ (with polar decomposition $x=v|x|$ ) and $w \in \mathcal{M}$ be given. Since $w v|x|^{1 / 2}$ and $|x|^{1 / 2}$ belong to $F$, it follows from (4.3) that

$$
\begin{aligned}
|\phi(w x)|^{2} & =\left|\phi\left(\left(|x|^{1 / 2} v^{*} w^{*}\right)^{*}|x|^{1 / 2}\right)\right| \\
& \leq \phi\left(\left(|x|^{1 / 2} v^{*} w^{*}\right)^{*}|x|^{1 / 2} v^{*} w^{*}\right) \phi(|x|) \\
& =\phi\left(w v|x| v^{*} w^{*}\right) \phi(|x|) .
\end{aligned}
$$

Since $v|x| v^{*}=\left|x^{*}\right|$, inequality (4.2) follows.

An immediate consequence of (4.2) is that

$$
|\phi(x)|^{2} \leq \phi\left(\left|x^{*}\right|\right) \phi(|x|), \quad x \in E,
$$

whenever $\phi \in\left(E^{*}\right)^{+}$. We wish to point out that the inequality $|\phi(x)| \leq \phi(|x|)$ is in general not valid for positive functionals, even in the special case that $\mathcal{M}$ is the von Neumann algebra $M_{2}(\mathbb{C})$ of all $2 \times 2$ matrices.

Corollary 4.3. If $\phi \in\left(E^{*}\right)^{+}$, then

$$
\|\phi\|_{E^{*}}=\sup \left\{\phi(x): x \in E^{+},\|x\|_{E} \leq 1\right\} .
$$

Consequently, $\|\phi\|_{E^{*}} \leq\|\psi\|_{E^{*}}$ whenever $0 \leq \phi \leq \psi$ in $E^{*}$.

Proof. Let $A$ denote the right hand side supremum. If $x \in E$ and $\|x\|_{E} \leq 1$, then $\||x|\|_{E}=\left\|\left|x^{*}\right|\right\|_{E} \leq 1$, and so it follows from (4.4) that $|\phi(x)|^{2} \leq A^{2}$. This suffices for the proof.

The following norm estimate will be used in Section 6

Lemma 4.4. If $x \in E^{+}$and $y \in \mathcal{M}$, then $\|x y\|_{E} \leq 4\|x\|_{E}^{1 / 2}\left\|y^{*} x y\right\|_{E}^{1 / 2}$.

Proof. Let $x y=v|x y|$ be the polar decomposition of $x y$. If $\phi \in\left(E^{*}\right)^{+}$satisfies $\|\phi\|_{E^{*}} \leq 1$, then using (4.3), it follows that

$$
\begin{aligned}
\phi(|x y|)^{2} & =\left|\phi\left(\left(x^{1 / 2} v\right)^{*}\left(x^{1 / 2} y\right)\right)\right|^{2} \leq \phi\left(v^{*} x v\right) \phi\left(y^{*} x y\right) \\
& \leq\|x\|_{E}\left\|y^{*} x y\right\|_{E} .
\end{aligned}
$$

Corollary 4.1 now yields the desired estimate.

It will be convenient to introduce the following notion. 
Definition 4.5. The absolute kernel $N(\phi)$ of a positive functional $\phi \in\left(E^{*}\right)^{+}$is defined by setting

$$
N(\phi)=\left\{x \in E: \phi(|x|)=\phi\left(\left|x^{*}\right|\right)=0\right\} .
$$

Using Lemma 4.2, the following properties of the absolute kernel are easily obtained.

Lemma 4.6. If $\phi \in\left(E^{*}\right)^{+}$, then

$$
N(\phi)=\{x \in E: \phi(w x)=\phi(x w)=0 \quad \forall w \in \mathcal{M}\} .
$$

We recall some definitions from [1].

Definition 4.7. (i) A linear subspace $J$ of $E$ is called an order ideal if $J$ is *-closed, $x \in J$ implies $|x| \in J$ and if $x \in E$ and $y \in J$ satisfy $0 \leq x \leq y$, then $x \in J$;

(ii) an ideal $J \subseteq E$ is called order dense (in $E$ ) if for every $0<x \in E^{+}$there exists $y \in J^{+}$such that $0<y \leq x$.

Note that any order ideal $J \subseteq E$ is generated by $J^{+}$as a linear subspace. Indeed, if $x \in J$, then $x^{*} \in J$, and so $\operatorname{Re} x$ and $\operatorname{Im} x$ belong to $J$. Moreover, if $a \in J$ and $a=a^{*}$, then $|a| \in J$ and $0 \leq a^{ \pm} \leq|a|$, which implies that $a^{ \pm} \in J$. It should also be observed that the intersection of order ideals is an order ideal. The intersection of finitely many order dense order ideals is also order dense. Evidently, the absolute kernel of a positive functional is an order ideal.

The following characterizations of order density are noted in [11] and are easily to proved.

Lemma 4.8. If $J \subseteq E$ is an order ideal, then the following statements are equivalent:

(i) $J$ is order dense;

(ii) for every $0<x \in E^{+}$there exists a family $\left\{x_{\alpha}: \alpha \in \mathbb{A}\right\}$ in $J^{+}$such that $x=\sum_{\alpha \in \mathbb{A}} x_{\alpha}$ (here, the sum $\sum_{\alpha \in \mathbb{A}} x_{\alpha}$ is interpreted as $\sup _{F} \sum_{\alpha \in F} x_{\alpha}$, where the supremum is taken over all finite subsets $F$ of $\mathbb{A}$ );

(iii) for every $0<x \in E^{+}$there exists an upwards directed net $\left\{x_{\alpha}\right\}$ in $J^{+}$such that $0 \leq x_{\alpha} \uparrow_{\alpha} x$;

(iv) for every $0<p \in P(E)$ there exists $q \in P(E) \cap J$ such that $0<q \leq p$.

Remark 4.9. Observe that an order ideal $J \subseteq E$ is order dense in $E$ if and only if $J \cap \mathcal{F}(\tau)$ is order dense in $\mathcal{F}(\tau)$ (assuming that $c_{E}=\mathbf{1}$, so that $\mathcal{F}(\tau) \subseteq E$ ).

Definition 4.10. A functional $\phi \in E^{*}$ is called

(i) normal if $\phi\left(x_{\alpha}\right) \rightarrow_{\alpha} 0$ whenever $x_{\alpha} \downarrow_{\alpha} 0$ in $E$;

(ii) completely additive if

$$
\phi\left(x\left(\sum_{i} e_{i}\right)\right)=\sum_{i} \phi\left(x e_{i}\right) \quad \text { and } \quad \phi\left(\left(\sum_{i} e_{i}\right) x\right)=\sum_{i} \phi\left(e_{i} x\right)
$$

for every collection $\left\{e_{i}\right\}$ of mutually orthogonal projections in $P(\mathcal{M})$ and every $x \in E$;

(iii) singular if $\phi$ vanishes on some order dense order ideal in $E$.

The sets of all normal and singular functionals on $E$ are denoted by $E_{n}^{*}$ and $E_{s}^{*}$, respectively. It is easy to see that $E_{n}^{*}$ and $E_{s}^{*}$ are linear subspaces of $E^{*}$. Furthermore, it follows immediately from the definitions that $E_{n}^{*} \cap E_{s}^{*}=\{0\}$. If 
$\phi \in E^{*}$ is normal, singular or completely additive, respectively, then $\bar{\phi}$ has the same property (consequently, the same holds for $\operatorname{Re} \phi$ and $\operatorname{Im} \phi$ ).

If $y \in E^{\times}$and the linear functional $\phi_{y} \in E^{*}$ is defined by $\phi_{y}(x)=\tau(x y), x \in E$, then $\phi_{y} \in E_{n}^{*}$ and $\left\|\phi_{y}\right\|_{E^{*}}=\|y\|_{E^{\times}}$(see [10], Theorem 5.11, implication (ii) $\Rightarrow($ iii)). Furthermore, the following observations should be made.

Lemma 4.11. If $y \in E^{\times}$, then:

(i) $w_{\alpha} \uparrow_{\alpha} w$ in $\mathcal{M}$ implies that $\phi_{y}\left(w_{\alpha} x\right) \rightarrow_{\alpha} \phi_{y}(w x)$ and $\phi_{y}\left(x w_{\alpha}\right) \rightarrow_{\alpha}$ $\phi_{y}(x w)$ for all $x \in E$;

(ii) the functional $\phi_{y}$ is completely additive.

Proof. (i) It should be observed first that $\tau\left(w_{\alpha} z\right) \rightarrow \tau(w z)$ whenever $w_{\alpha} \uparrow_{\alpha} w$ in $\mathcal{M}$ and $z \in L_{1}(\tau)$. Indeed, without loss of generality, it may be assumed that $z \geq 0$. In this case, $z^{1 / 2} w_{\alpha} z^{1 / 2} \uparrow_{\alpha} z^{1 / 2} w z^{1 / 2}$, and so

$$
\tau\left(w_{\alpha} z\right)=\tau\left(z^{1 / 2} w_{\alpha} z^{1 / 2}\right) \uparrow_{\alpha} \tau\left(z^{1 / 2} w z^{1 / 2}\right)=\tau(w z),
$$

which proves the claim. Consequently, if $y \in E^{\times}$and $x \in E$, then $x y \in L_{1}(\tau)$, and hence

$$
\phi_{y}\left(w_{\alpha} x\right)=\tau\left(w_{\alpha} x y\right) \rightarrow_{\alpha} \tau(w x y)=\phi_{y}(w x)
$$

whenever $w_{\alpha} \uparrow_{\alpha} w$ in $\mathcal{M}$. Since $\phi_{y}\left(x w_{\alpha}\right)=\overline{\phi_{y^{*}}\left(w_{\alpha} x^{*}\right)}$, the second assertion of (i) is now also clear.

(ii) This follows immediately from (i).

It should be recalled that the norm $\|\cdot\|_{E}$ is called order continuous if $\left\|x_{\alpha}\right\|_{E} \downarrow_{\alpha} 0$ for every downwards directed system $\left\{x_{\alpha}\right\} \subseteq E^{+}$satisfying $x_{\alpha} \downarrow_{\alpha} 0$ (equivalently, $\left\|x_{n}\right\|_{E} \downarrow 0$ as $n \rightarrow \infty$ for every downwards directed sequence $\left\{x_{n}\right\}_{n=1}^{\infty} \subseteq E^{+}$ satisfying $x_{n} \downarrow_{n} 0$ ). Using Mazur's theorem, the following observation is easily established.

Proposition 4.12. The norm in $E$ is order continuous if and only if $E^{*}=E_{n}^{*}$.

Now, consider the special case that $E=\mathcal{M}$ and denote by $\mathcal{M}_{*}$ the subspace of $\mathcal{M}^{*}$ consisting of all ultra-weakly continuous linear functionals on $\mathcal{M}$, which may be identified with the predual of $\mathcal{M}$ (see [6], Theorem I.3.1). Using [26, Theorems III.2.14 and III.3.8, in combination with Lemma 4.8, it may be shown that $\mathcal{M}_{n}^{*}=\mathcal{M}_{*}$ and that $\mathcal{M}_{s}^{*}$ coincides with the subspace of all singular functionals as defined in Definition III.2.13 (see also [18, Section 10.1). We collect these observations in the next theorem (see [18, Theorems 10.1.15 and 10.1.19, 26], Theorem III.2.14 and [6], Section I.6.10).

Theorem 4.13. $\quad$ (i) Every $\phi \in \mathcal{M}^{*}$ has a unique decomposition $\phi=\phi_{n}+\phi_{s}$ with $\phi_{n} \in \mathcal{M}_{n}^{*}$ and $\phi_{s} \in \mathcal{M}_{s}^{*}$. If $\phi \geq 0$, then $\phi_{n} \geq 0$ and $\phi_{s} \geq 0$.

(ii) If $\phi \in \mathcal{M}_{s}^{*}$ (respectively, $\phi \in \mathcal{M}_{n}^{*}$ ), then $x \phi, \phi x \in \mathcal{M}_{s}^{*}$ (respectively, $x \phi, \phi x \in \mathcal{M}_{n}^{*}$ ) for all $x \in \mathcal{M}$.

(iii) A linear functional $\phi$ on $\mathcal{M}$ belongs to $\mathcal{M}_{n}^{*}$ if and only if there exists a (unique) $y \in L_{1}(\tau)$ such that $\phi(x)=\tau(x y), x \in \mathcal{M}$. Moreover, in this case, $\phi$ is hermitian (respectively, positive) if and only if $y=y^{*}$ (respectively, $y \geq 0$ ).

(iv) A linear functional $\phi \in \mathcal{M}^{*}$ is normal if and only if $\phi$ is completely additive on projections (that is, $\phi\left(\sum_{\alpha} e_{\alpha}\right)=\sum_{\alpha} \phi\left(e_{\alpha}\right)$ whenever $\left\{e_{\alpha}\right\}$ is a mutually orthogonal family in $P(\mathcal{M})$ ). 


\section{THE DECOMPOSITION THEOREM}

Let $\mathcal{M}$ be a von Neumann algebra on a Hilbert space $H$, equipped with a normal semi-finite faithful trace $\tau$. In this section it will be shown that every bounded linear functional on a strongly symmetrically normed space $E \subseteq S(\tau)$ has a unique decomposition in a normal and a singular part. Moreover, the space of normal functionals will be identified with the Köthe dual space. The proof of these results (see Theorems 5.8 and 5.9) is divided into a number of lemmas and propositions.

Lemma 5.1. If for every $e \in P_{f}(\mathcal{M})$ there exists $a_{e} \in L_{1}(\tau)$ such that:

(i) $a_{e}=a_{f}$ e whenever $e, f \in P_{f}(\mathcal{M})$ satisfy $e \leq f$;

(ii) for all $e \in P_{f}(\mathcal{M})$, the operator $e a_{e}$ is self-adjoint,

then there exists a closed, densely defined symmetric operator a in $H$, which is affiliated with $\mathcal{M}$, such that ae $=a_{e}$ for $e \in P_{f}(\mathcal{M})$.

Proof. Observing that (i) implies, in particular, that $a_{e}=a_{e} e$ for all $e \in P_{f}(\mathcal{M})$, it follows from (i) that

$$
\mathfrak{D}\left(a_{e}\right) \cap e(H)=\mathfrak{D}\left(a_{f}\right) \cap e(H) \subseteq \mathfrak{D}\left(a_{f}\right) \cap f(H)
$$

whenever $e \leq f$ in $P_{f}(\mathcal{M})$. Since $P_{f}(\mathcal{M})$ is upwards directed, this shows that

$$
\left\{\mathfrak{D}\left(a_{e}\right) \cap e(H): e \in P_{f}(\mathcal{M})\right\}
$$

is an upwards directed system of linear subspaces of $H$, with respect to inclusion. Therefore,

$$
\mathfrak{D}\left(a_{0}\right)=\bigcup\left\{\mathfrak{D}\left(a_{e}\right) \cap e(H): e \in P_{f}(\mathcal{M})\right\}
$$

is a linear subspace of $H$. If $e, f \in P_{f}(\mathcal{M})$ are such that $e \leq f$, and if $\xi \in$ $\mathfrak{D}\left(a_{e}\right) \cap e(H)$, then it follows from (i) that $a_{e} \xi=a_{f} e \xi=a_{f} \xi$, and so the linear operator $a_{0}: \mathfrak{D}\left(a_{0}\right) \rightarrow H$ may be defined by setting

$$
a_{0} \xi=a_{e} \xi, \quad \xi \in \mathfrak{D}\left(a_{e}\right) \cap e(H) \text { for some } e \in P_{f}(\mathcal{M})
$$

Using the fact that for each $e \in P_{f}(\mathcal{M})$ the domain $\mathfrak{D}\left(a_{e}\right)$ of $a_{e}$ is dense in $H$, it follows that $\mathfrak{D}\left(a_{e}\right) \cap e(H)$ is dense in $e(H)$. Since the trace $\tau$ is semi-finite, the linear subspace

$$
\bigcup\left\{e(H): e \in P_{f}(\mathcal{M})\right\}
$$

is dense in $H$, and hence $\mathfrak{D}\left(a_{0}\right)$ is dense in $H$.

Since the collection (5.1) is upwards directed, given $\xi, \eta \in \mathfrak{D}\left(a_{0}\right)$, there exists an $e \in P_{f}(\mathcal{M})$ such that $\xi, \eta \in \mathfrak{D}\left(a_{e}\right) \cap e(H)$. Therefore, assumption (ii) easily implies that $a_{0}$ is symmetric. Consequently, $a_{0} \subseteq a_{0}^{*}$, and hence, in particular, the operator $a_{0}$ is preclosed. Furthermore, since $a_{e} \in L_{1}(\tau)$ implies, in particular, that $a_{e} \eta \mathcal{M}$ for all $e \in P_{f}(\mathcal{M})$, it is easy to verify that $a_{0} \eta \mathcal{M}$.

It should be noted next that $a_{0} e=a_{e}$ whenever $e \in P_{f}(\mathcal{M})$. Indeed, if $\xi \in$ $\mathfrak{D}\left(a_{e}\right)$, then $e \xi \in \mathfrak{D}\left(a_{e}\right) \cap e(H) \subseteq \mathfrak{D}\left(a_{0}\right)$ and $a_{0} e \xi=a_{e} e \xi=a_{e} \xi$. This shows that $a_{e} \subseteq a_{0} e$. Conversely, if $\xi \in \mathfrak{D}\left(a_{0} e\right)$, then $e \xi \in \mathfrak{D}\left(a_{0}\right)$, and so there exists $f \in P_{f}(\mathcal{M})$ such that $e \xi \in \mathfrak{D}\left(a_{f}\right) \cap f(H)$. Since (5.1) is an upwards directed system, it may be assumed, without loss of generality, that $e \leq f$. Consequently, $e \xi \in \mathfrak{D}\left(a_{f}\right) \cap e(H)=\mathfrak{D}\left(a_{e}\right) \cap e(H)$, which implies that $\xi \in \mathfrak{D}\left(a_{e}\right)$ and $a_{0} e \xi=$ $a_{e} e \xi=a_{e} \xi$. Hence, $a_{0} e \subseteq a_{e}$, and it may be concluded that $a_{0} e=a_{e}$.

Let $a$ be the closure of the operator $a_{0}$. Evidently, $a$ is densely defined and symmetric, and $a$ is affiliated with $\mathcal{M}$. It remains to be shown that $a e=a_{e}$ whenever $e \in P_{f}(\mathcal{M})$. To this end, observe that ae is closed, that ae $\mathcal{M}$ and that 
$a_{e}=a_{0} e \subseteq a e$. Since $a_{e} \in L_{1}(\tau)$, the operator $a_{e}$ is $\tau$-measurable, and so, in particular, its domain $\mathfrak{D}\left(a_{e}\right)$ is $\tau$-dense in $H$. It follows from $\mathfrak{D}\left(a_{e}\right) \subseteq \mathfrak{D}(a e)$ that $\mathfrak{D}(a e)$ is also $\tau$-dense, and hence ae is $\tau$-measurable. Therefore (see e.g. [28]), $a_{e}=a e$. This completes the proof of the lemma.

Recall that $\mathcal{F}(\tau)$, as defined by (2.2), is a strongly symmetrically normed space, equipped with the norm $\|\cdot\|_{\mathcal{F}(\tau)}=\|\cdot\|_{L_{1} \cap L_{\infty}}$.

Lemma 5.2. If $\psi$ is a bounded hermitian linear functional on $\mathcal{F}(\tau)$, then there exists a closed symmetric densely defined operator a in $H$, affiliated with $\mathcal{M}$, such that:

(i) ae $\in L_{1}(\tau)$ for each $e \in P_{f}(\mathcal{M})$;

(ii) for each $e \in P_{f}(\mathcal{M})$ there exists a singular functional $\psi_{s}^{e} \in \mathcal{M}_{s}^{*}$ such that

$$
\psi(e z)=\tau(z a e)+\psi_{s}^{e}(z), \quad z \in \mathcal{M} .
$$

Proof. For $e \in P_{f}(\mathcal{M})$, define the linear functional $\psi^{e}$ on $\mathcal{M}$ by setting

$$
\psi^{e}(z)=\psi(e z), \quad z \in \mathcal{M} .
$$

Since

$$
\left|\psi^{e}(z)\right|=|\psi(e z)| \leq\|\psi\|_{\mathcal{F}(\tau)^{*}}\|e z\|_{\mathcal{F}(\tau)} \leq\|\psi\|_{\mathcal{F}(\tau)^{*}}\|e\|_{\mathcal{F}(\tau)}\|z\|_{\infty}, \quad z \in \mathcal{M},
$$

it is clear that $\psi^{e} \in \mathcal{M}^{*}$. Therefore, by Theorem 4.13 (i), $\psi^{e}$ has a unique decomposition into its normal and singular parts; that is,

$$
\psi^{e}=\psi_{n}^{e}+\psi_{s}^{e}, \quad \psi_{n}^{e} \in \mathcal{M}_{*}, \psi_{s}^{e} \in \mathcal{M}_{s}^{*} .
$$

Furthermore, by Theorem 4.13 (iii), there exists a unique $a_{e} \in L_{1}(\tau)$ such that $\psi_{n}^{e}(z)=\tau\left(z a_{e}\right), z \in \mathcal{M}$. Consequently,

$$
\psi(e z)=\tau\left(z a_{e}\right)+\psi_{s}^{e}(z), \quad z \in \mathcal{M} .
$$

Observe first that $a_{e}=a_{f} e$ whenever $e, f \in P_{f}(\mathcal{M})$ satisfy $e \leq f$. Indeed, if this is the case, then

$$
\begin{aligned}
\psi^{e}(z) & =\psi(e z)=\psi(f e z)=\tau\left(e z a_{f}\right)+\psi_{s}^{f}(e z) \\
& =\tau\left(z a_{f} e\right)+\left(\psi_{s}^{f} e\right)(z), \quad z \in \mathcal{M} .
\end{aligned}
$$

Since the functional $\psi_{s}^{f} e$ is singular on $\mathcal{M}$ (see Theorem 4.13 (ii)), it follows from the uniqueness of the decomposition in (5.2) that $a_{e}=a_{f} e$ in $L_{1}(\tau)$.

It will be shown next that $e a_{e}$ is self-adjoint for all $e \in P_{f}(\mathcal{M})$. To this end, observe that

$$
\psi(e z e)=\tau\left(z e a_{e}\right)+\psi_{s}^{e}(z e), \quad z \in \mathcal{M} .
$$

Taking complex conjugates in the above identity and using the fact that $\psi$ is hermitian, this implies that

$$
\begin{aligned}
\psi\left(e z^{*} e\right) & =\tau\left(z^{*}\left(e a_{e}\right)^{*}\right)+\overline{\psi_{s}^{e}(z e)} \\
& =\tau\left(z^{*}\left(e a_{e}\right)^{*}\right)+\overline{\psi_{s}^{e}}\left(e z^{*}\right), \quad z \in \mathcal{M},
\end{aligned}
$$

that is,

$$
\psi(e z e)=\tau\left(z\left(e a_{e}\right)^{*}\right)+\overline{\psi_{s}^{e}}(e z), \quad z \in \mathcal{M} .
$$

Consequently,

$$
\tau\left(z e a_{e}\right)-\tau\left(z\left(e a_{e}\right)^{*}\right)=\overline{\psi_{s}^{e}}(e z)-\psi_{s}^{e}(z e), \quad z \in \mathcal{M} .
$$


Since the functional $z \longmapsto \tau\left(z e a_{e}\right)-\tau\left(z\left(e a_{e}\right)^{*}\right), z \in \mathcal{M}$, is normal and the functional $z \longmapsto \overline{\psi_{s}^{e}}(e z)-\psi_{s}^{e}(z e), z \in \mathcal{M}$, is singular on $\mathcal{M}$, this implies that

$$
\tau\left(z e a_{e}\right)=\tau\left(z\left(e a_{e}\right)^{*}\right), \quad z \in \mathcal{M}
$$

and hence, $e a_{e}=\left(e a_{e}\right)^{*}$, that is, $e a_{e}$ is self-adjoint.

It now follows from Lemma 5.1 that there exists a closed, densely defined symmetric operator $a$ in $H$, affiliated with $\mathcal{M}$, such that $a_{e}=a e$ for all $e \in P_{f}(\mathcal{M})$. The proof of the lemma is complete.

The following lemma is needed to show that the operator $a$, occurring in the above lemma, actually belongs to $S(\tau)$ and is self-adjoint.

Lemma 5.3. Let $b$ be a positive self-adjoint operator, affiliated with $\mathcal{M}$, such that be $\in L_{1}(\tau)$ for all $e \in P_{f}(\mathcal{M})$. If there exists a constant $C \geq 0$ such that

$$
\tau(b e) \leq C\|e\|_{\mathcal{F}(\tau)}, \quad e \in P_{f}(\mathcal{M}),
$$

then $b \in S(\tau)$.

Proof. It should be observed first that ebe $\geq 0$, and so $\tau(b e)=\tau(e b e) \geq 0$ for all $e \in P_{f}(\mathcal{M})$. If $e \in P_{f}(\mathcal{M})$ satisfies $\tau(e) \geq 1$, then $\|e\|_{\mathcal{F}(\tau)}=\tau(e)$, and so it follows from (5.3) that

$$
\tau(b e) \leq C \tau(e), \quad e \in P_{f}(\mathcal{M}), \tau(e) \geq 1 .
$$

Suppose that $b$ is not $\tau$-measurable, that is, $\tau\left(e^{b}(\lambda, \infty)\right)=\infty$ for all $\lambda>0$. This implies that there exists a strictly increasing sequence $\left\{\lambda_{n}\right\}_{n=1}^{\infty}$ in $[0, \infty)$ such that $\lambda_{n} \uparrow \infty$ and $\tau\left(e^{b}\left(\lambda_{n}, \lambda_{n+1}\right]\right)>1$ for all $n$. Since the trace $\tau$ is semi-finite, there exists for each $n$ a projection $e_{n} \in P_{f}(\mathcal{M})$ which satisfies $e_{n} \leq$ $e^{b}\left(\lambda_{n}, \lambda_{n+1}\right]$ and $\tau\left(e_{n}\right) \geq 1$. It follows from $b e^{b}\left(\lambda_{n}, \lambda_{n+1}\right] \geq \lambda_{n} e^{b}\left(\lambda_{n}, \lambda_{n+1}\right]$ that $e_{n} b e^{b}\left(\lambda_{n}, \lambda_{n+1}\right] e_{n} \geq \lambda_{n} e_{n}$, that is, $e_{n} b e_{n} \geq \lambda_{n} e_{n}$ for all $n$. Consequently,

$$
\lambda_{n} \tau\left(e_{n}\right) \leq \tau\left(e_{n} b e_{n}\right)=\tau\left(b e_{n}\right) \leq C \tau\left(e_{n}\right)
$$

for all $n$. This is absurd, and so it may be concluded that $b$ is $\tau$-measurable.

Lemma 5.4. Suppose that $E \subseteq S(\tau)$ is a strongly symmetrically normed space (with $c_{E}=1$ ). If $\psi$ is a hermitian bounded linear functional on $E$ and if $a$ is a closed symmetric operator in $H$, affiliated with $\mathcal{M}$, satisfying the conclusion of Lemma [5.2, then:

(i) $|\tau(z a e)| \leq\|\psi\|_{E^{*}}\|z\|_{E}$ for all $z \in \mathcal{F}(\tau)^{+}$and all $e \in P_{f}(\mathcal{M})$;

(ii) $\tau(|z a e|) \leq 4\|\psi\|_{E^{*}}\|z\|_{E}$ for all $z \in \mathcal{F}(\tau)$ and all $e \in P_{f}(\mathcal{M})$;

(iii) $|a| e \in L_{1}(\tau)$ and $0 \leq \tau(|a| e) \leq 4\|\psi\|_{E^{*}}\|e\|_{E}$ for all $e \in P_{f}(\mathcal{M})$;

(iv) the operator a is $\tau$-measurable and self-adjoint.

Proof. It should be observed first that $\mathcal{F}(\tau)$ is embedded continuously in $E$ (see the remarks made in Section 3 ). Therefore, the restriction of $\psi$ to $\mathcal{F}(\tau)$ is bounded. Consequently, by Lemma 5.2. there exists a closed symmetric operator $a$ in $H$ which satisfies the conclusion of that lemma.

(i) If $e \in P_{f}(\mathcal{M})$, then

$$
\psi(e z)=\tau(z a e)+\psi_{s}^{e}(z), \quad z \in \mathcal{M}
$$

where the functional $\psi_{s}^{e}$ is singular on $\mathcal{M}$. The functional $\psi_{s}^{e}$ vanishes on some order dense order ideal $J \subseteq \mathcal{M}$, and so

$$
|\tau(z a e)|=|\psi(e z)| \leq\|\psi\|_{E^{*}}\|e z\|_{E} \leq\|\psi\|_{E^{*}}\|z\|_{E}, \quad z \in J
$$


Suppose now that $0 \leq z \in \mathcal{F}(\tau)$. Since $J$ is order dense, Lemma 4.8 implies that there exists an upwards directed system $\left\{z_{\alpha}\right\}$ in $J^{+}$such that $0 \leq z_{\alpha} \uparrow_{\alpha} z$. Since $a e \in L_{1}(\tau) \subseteq S_{0}(\tau)$, it follows from Lemma 2.2 that $z_{\alpha} a e \stackrel{\mathcal{T}_{r_{2}}}{\rightarrow_{\alpha}} z a e$, and so there exists a sequence $\alpha_{1} \leq \alpha_{2} \leq \cdots$ such that $z_{\alpha_{n}}$ ae $\stackrel{\mathcal{T}_{m}}{\rightarrow} z a e$ as $n \rightarrow \infty$. Since $\mu\left(z_{\alpha_{n}} a e\right) \leq \mu(a e) \in L_{1}(\tau)$ for all $n$, it follows from the non-commutative version of the dominated convergence theorem (see e.g. [14, Theorem 3.5) that $\tau\left(z_{\alpha_{n}} a e\right) \rightarrow$ $\tau(z a e)$ as $n \rightarrow \infty$. For each $n$, it follows from (5.4) that $\left|\tau\left(z_{\alpha_{n}} a e\right)\right| \leq\|\psi\|_{E^{*}}\|z\|_{E}$, and hence $|\tau(z a e)| \leq\|\psi\|_{E^{*}}\|z\|_{E}$.

(ii) If $z \in \mathcal{F}(\tau)$, then $\left\|(\operatorname{Re} z)^{ \pm}\right\|_{E} \leq\|z\|_{E}$ and $\left\|(\operatorname{Im} z)^{ \pm}\right\|_{E} \leq\|z\|_{E}$. Therefore, it follows immediately from (i) that $|\tau(z a e)| \leq 4\|\psi\|_{E^{*}}\|z\|_{E}$. If $z a e=v|z a e|$ is the polar decomposition of $z a e$, then $|z a e|=v^{*} z a e$, and hence

$$
\tau(|z a e|)=\tau\left(v^{*} z a e\right) \leq 4\|\psi\|_{E^{*}}\left\|v^{*} z\right\|_{E} \leq 4\|\psi\|_{E^{*}}\|z\|_{E} .
$$

(iii) Let $a=v|a|$ be the polar decomposition of the operator $a$ (which exists since $a$ is a closed and densely defined operator) and suppose that $e \in P_{f}(\mathcal{M})$. Since $|a|=v^{*} a$, it is clear that $|a| e=v^{*} a e \in L_{1}(\tau)$. Since $e|a| e \geq 0$, it is also clear that $\tau(|a| e)=\tau(e|a| e) \geq 0$. It now follows from the estimate in (ii) that

$$
\begin{aligned}
\tau(|a| e) & =\tau\left(v^{*} a e\right)=\tau\left(e v^{*} a e\right) \leq \tau\left(\left|e v^{*} a e\right|\right) \\
& \leq 4\|\psi\|_{E^{*}}\left\|e v^{*}\right\|_{E} \leq 4\|\psi\|_{E^{*}}\|e\|_{E} .
\end{aligned}
$$

(iv) Since $\mathcal{F}(\tau)$ is continuously embedded in $E$, there exists a constant $C>0$ such that $\|x\|_{E} \leq C\|x\|_{\mathcal{F}(\tau)}$ for all $x \in \mathcal{F}(\tau)$. Therefore, it follows from (iii) that

$$
\tau(|a| e) \leq 4\|\psi\|_{E^{*}}\|e\|_{E} \leq 4 C\|\psi\|_{E^{*}}\|e\|_{\mathcal{F}(\tau)}, \quad e \in P_{f}(\mathcal{M}) .
$$

By Lemma [5.3 this implies that $|a| \in S(\tau)$. Consequently, $a=v|a| \in S(\tau)$. Since $a$ is symmetric, this yields, in particular, that $a$ is self-adjoint (see [28]).

Proposition 5.5. Let $E \subseteq S(\tau)$ be a strongly symmetrically normed space (with $c_{E}=1$ ). If $\psi \in E^{*}$ is hermitian, then there exists a unique $a \in E^{\times}$such that for each $e \in P_{f}(\mathcal{M})$ there exists a singular functional $\psi_{s}^{e} \in \mathcal{M}_{s}^{*}$ satisfying

$$
\psi(e z)=\tau(z a e)+\psi_{s}^{e}(z), \quad z \in \mathcal{M} .
$$

Moreover, the operator $a$ is self-adjoint. Furthermore, if $\psi \geq 0$, then $a \geq 0$ and $e \psi_{s}^{e} \geq 0$ for all $e \in P_{f}(\mathcal{M})$.

Proof. First observe that it follows from Lemmas 5.2 and 5.4 (iv) that there exists a self-adjoint operator $a \in S(\tau)$ such that $a e \in L_{1}(\tau)$ for all $e \in P_{f}(\mathcal{M})$ and (5.5) is satisfied. If $e \in P_{f}(\mathcal{M})$, then it follows from Lemma 5.4 (ii) that

$$
\sup \left\{\tau(|z a e|): z \in \mathcal{F}(\tau),\|z\|_{E} \leq 1\right\} \leq 4\|\psi\|_{E^{*}}<\infty .
$$

By Remark 3.6 (i), this implies that $a e \in E^{\times}$and $\|a e\|_{E^{\times}} \leq 4\|\psi\|_{E^{*}}$. If $a=v|a|$ is the polar decomposition of $a$, then $e|a| e=e v^{*} a e$, which implies that $e|a| e \in E^{\times}$ and $\|e|a| e\|_{E^{\times}} \leq 4\|\psi\|_{E^{*}}$ for all $e \in P_{f}(\mathcal{M})$. Let $\left\{e_{\alpha}\right\}$ be an upwards directed system in $P_{f}(\mathcal{M})$ such that $e_{\alpha} \uparrow \mathbf{1}$. Since

$$
\begin{aligned}
\mu\left(|a|^{1 / 2} e_{\alpha}|a|^{1 / 2}\right) & =\mu\left(\left(e_{\alpha}|a|^{1 / 2}\right)^{*} e_{\alpha}|a|^{1 / 2}\right) \\
& =\mu\left(e_{\alpha}|a|^{1 / 2}\left(e_{\alpha}|a|^{1 / 2}\right)^{*}\right)=\mu\left(e_{\alpha}|a| e_{\alpha}\right),
\end{aligned}
$$


it follows that $|a|^{1 / 2} e_{\alpha}|a|^{1 / 2} \in E^{\times}$and

$$
\left\||a|^{1 / 2} e_{\alpha}|a|^{1 / 2}\right\|_{E^{\times}}=\left\|e_{\alpha}|a| e_{\alpha}\right\|_{E^{\times}} \leq 4\|\psi\|_{E^{*}}
$$

for all $\alpha$. Using the fact that $|a|^{1 / 2} e_{\alpha}|a|^{1 / 2} \uparrow_{\alpha}|a|$ (see [10, Proposition 1.4) and that $E^{\times}$has the Fatou property (see Proposition 3.7), we may conclude that $|a| \in E^{\times}$and $\||a|\|_{E^{\times}} \leq 4\|\psi\|_{E^{*}}$, that is, $a \in E^{\times}$and $\|a\|_{E^{\times}} \leq 4\|\psi\|_{E^{*}}$.

To prove the uniqueness of the operator $a$, suppose that $b \in E^{\times}$is such that

$$
\psi(e z)=\tau(z b e)+\psi_{s}^{e}(z), \quad z \in \mathcal{M},
$$

for all $e \in P_{f}(\mathcal{M})$. This implies that

$$
\tau(z b e)=\tau(z a e), \quad z \in \mathcal{M},
$$

and since $b e$ and $a e$ both belong to $L_{1}(\tau)$, it follows that $b e=a e$ for all $e \in P_{f}(\mathcal{M})$. Let $\left\{e_{\alpha}\right\}$ be a net in $P_{f}(\mathcal{M})$ such that $e_{\alpha} \uparrow_{\alpha} \mathbf{1}$. This implies that $e_{\alpha} \rightarrow \mathbf{1}$ locally in measure, and so $e_{\alpha}(b-a) \rightarrow b-a$ locally in measure. Consequently, $b-a=0$, that is, $b=a$. This proves the uniqueness statement of the proposition.

Assume now that $0 \leq \psi \in E^{*}$. If $e \in P_{f}(\mathcal{M})$, then

$$
\psi(e z e)=\tau(z e a e)+\psi_{s}^{e}(z e)=\tau(z e a e)+\left(e \psi_{s}^{e}\right)(z), \quad z \in \mathcal{M},
$$

and the functional $z \longmapsto \psi(e z e), z \in \mathcal{M}$, is positive. The functional $z \longmapsto \tau(z e a e)$, $z \in \mathcal{M}$, is normal, and $e \psi_{s}^{e}$ is singular (see Theorem 4.13 (ii)). Since the normal and singular parts of a positive functional in $\mathcal{M}^{*}$ are both positive (see Theorem 4.13 (i)), this implies that $\tau(z e a e) \geq 0$ for all $z \in \mathcal{M}^{+}$and $e \psi_{s}^{e} \geq 0$. Hence, eae $\geq 0$ in $L_{1}(\tau)$ for all $e \in P_{f}(\mathcal{M})$. If $e \in P(\mathcal{M})$ is such that $e \leq s\left(a^{-}\right)$, then eae $=-e a^{-} e \leq 0$. Hence, $e a^{-} e=0$, equivalently, $\left(a^{-}\right)^{1 / 2} e\left(a^{-}\right)^{1 / 2}=0$ for all $e \in P_{f}(\mathcal{M})$ satisfying $e \leq s\left(a^{-}\right)$. If $\left\{e_{\alpha}\right\}$ is an increasing system in $P_{f}(\mathcal{M})$ such that $e_{\alpha} \uparrow_{\alpha} s\left(a^{-}\right)$, then $\left(a^{-}\right)^{1 / 2} e_{\alpha}\left(a^{-}\right)^{1 / 2} \uparrow_{\alpha}\left(a^{-}\right)^{1 / 2} s\left(a^{-}\right)\left(a^{-}\right)^{1 / 2}$, and so $\left(a^{-}\right)^{1 / 2} s\left(a^{-}\right)\left(a^{-}\right)^{1 / 2}=0$, that is, $a^{-}=s\left(a^{-}\right) a^{-} s\left(a^{-}\right)=0$. Consequently, $a \geq 0$. The proof of the proposition is complete.

Proposition 5.6. Let $E \subseteq S(\tau)$ be a strongly symmetrically normed space (with $\left.c_{E}=1\right)$. If $0 \leq \psi \in E^{*}$, then $\psi$ admits a unique decomposition

$$
\psi=\psi_{n}+\psi_{s}
$$

where $\psi_{n} \in E_{n}^{*}$ and $\psi_{s} \in E_{s}^{*}$. Moreover, $\psi_{n} \geq 0$ and $\psi_{s} \geq 0$, and there exists a unique $0 \leq a \in E^{\times}$such that $\psi_{n}(x)=\tau(x a), x \in E$.

Proof. Since $\psi \geq 0$ implies, in particular, that $\psi$ is hermitian, Proposition 5.5 is applicable. Consequently, there exists a unique element $0 \leq a \in E^{\times}$such that for each $e \in P_{f}(\mathcal{M})$, there exists a singular functional $\psi_{s}^{e} \in \mathcal{M}_{s}^{*}$ satisfying

$$
\psi(e z)=\tau(z a e)+\psi_{s}^{e}(z), \quad z \in \mathcal{M} .
$$

Define $0 \leq \psi_{n} \in E^{*}$ by setting

$$
\psi_{n}(x)=\tau(x a), \quad x \in E,
$$

and let $\psi_{s} \in E^{*}$ be defined by

$$
\psi_{s}=\psi-\psi_{n}
$$


As observed in Section 4, the functional $\psi_{n}$ is normal. The first objective is to show that $\psi_{s}$ is singular. If $e \in P_{f}(\mathcal{M})$ and $z \in \mathcal{M}$, then

$$
\begin{aligned}
\psi_{s}(e z) & =\psi(e z)-\tau(e z a) \\
& =\tau(z a e)+\psi_{s}^{e}(z)-\tau(e z a)=\psi_{s}^{e}(z) .
\end{aligned}
$$

Consequently,

$$
\psi_{s}(e z e)=\psi_{s}^{e}(z e)=\left(e \psi_{s}^{e}\right)(z), \quad z \in \mathcal{M}, \quad e \in P_{f}(\mathcal{M}) .
$$

By Proposition [5.5 this implies that $\psi_{s}(e z e) \geq 0$ whenever $z \in \mathcal{M}^{+}$and $e \in$ $P_{f}(\mathcal{M})$. Since

$$
\mathcal{F}(\tau)=\bigcup\left\{e \mathcal{M} e: e \in P_{f}(\mathcal{M})\right\}
$$

this implies that $\psi_{s}$ is a positive functional on $\mathcal{F}(\tau)$. Therefore,

$$
J_{0}=\left\{z \in \mathcal{F}(\tau): \psi_{s}(|z|)=\psi_{s}\left(\left|z^{*}\right|\right)=0\right\},
$$

which is the absolute kernel of $\left.\psi_{s}\right|_{\mathcal{F}(\tau)}$, is an order ideal in $\mathcal{F}(\tau)$ (and hence, in $E$ ). It should be observed that $J_{0}$ is order dense. Indeed, if $0<e \in P_{f}(\mathcal{M})$, then the functional $e \psi_{s}^{e}$ is singular on $\mathcal{M}$, and so there exists $f \in P(\mathcal{M})$ satisfying $0<f \leq e$ and $\left(e \psi_{s}^{e}\right)(f)=0$. Hence, it follows from (5.7) that

$$
\psi_{s}(f)=\psi_{s}(e f e)=\left(e \psi_{s}^{e}\right)(f)=0,
$$

and so $f \in J_{0}$. Therefore, since $\tau$ is semi-finite, it may be concluded, via Lemma 4.8, that $J_{0}$ is order dense in $E$. Consequently, $\psi_{s}$ is singular.

It will be shown next that $\psi_{s} \geq 0$. If $x \in E^{+}$, then, by Lemma 4.8, there exists an upwards directed system $\left\{x_{\alpha}\right\}$ in $J_{0}^{+}$such that $0 \leq x_{\alpha} \uparrow_{\alpha} x$. Since $\psi_{s}\left(x_{\alpha}\right)=0$, it follows that

$$
\begin{aligned}
\psi_{s}(x) & =\psi_{s}\left(x-x_{\alpha}\right)=\psi\left(x-x_{\alpha}\right)-\psi_{n}\left(x-x_{\alpha}\right) \\
& \geq-\psi_{n}\left(x-x_{\alpha}\right)
\end{aligned}
$$

for all $\alpha$. The normality of $\psi_{n}$ implies that $\psi_{n}\left(x-x_{\alpha}\right) \downarrow_{\alpha} 0$, and so $\psi_{s}(x) \geq 0$.

Finally, as already has been observed in Section $4, E_{n}^{*} \cap E_{s}^{*}=\{0\}$, and so the uniqueness of the decomposition is evident.

An interesting consequence of Proposition 5.6 is the following characterization of positive singular functionals. For the case $E=\mathcal{M}$ see e.g. [18], Proposition 10.1.17 (i).

Corollary 5.7. If $E \subseteq S(\tau)$ is a strongly symmetrically normed space, then a functional $0 \leq \psi \in E^{*}$ is singular if and only if $\psi$ does not majorize any non-zero positive normal functional.

Proof. Suppose that $0 \leq \psi \in E_{s}^{*}$ and $0 \leq \phi \in E_{n}^{*}$ such that $0 \leq \phi \leq \psi$. By definition, $\psi$ vanishes on some order dense order ideal $J \subseteq E$. If $x \in J^{+}$, then $0 \leq \phi(x) \leq \psi(x)=0$, and so $\phi(x)=0$. Since $J$ is generated by $J^{+}$, it follows that $\phi$ vanishes on $J$. Hence, $\phi$ is singular. Since $E_{n}^{*} \cap E_{s}^{*}=\{0\}$, this implies that $\phi=0$.

Suppose now that $\psi$ does not majorize any non-zero positive normal functional. By Proposition 5.6, there exist $0 \leq \psi_{n} \in E_{n}^{*}$ and $0 \leq \psi_{s} \in E_{s}^{*}$ such that $\psi=\psi_{n}+\psi_{s}$. Since $0 \leq \psi_{n} \leq \psi$, it follows that $\psi_{n}=0$, and so $\psi=\psi_{s}$. The proof is complete.

The following theorem is the main result of the present paper. 
Theorem 5.8. Let $E \subseteq S(\tau)$ be a strongly symmetrically space normed (with $\left.c_{E}=1\right)$. Every $\psi \in E^{*}$ admits a unique decomposition

$$
\psi=\psi_{n}+\psi_{s}
$$

where $\psi_{s} \in E_{s}^{*}$ and $\psi_{n} \in E_{n}^{*}$ is given by $\psi_{n}(x)=\tau(x y), x \in E$, for a unique $y \in E^{\times}$. Moreover, if $\psi$ is hermitian, then $y=y^{*}$, and if $\psi \geq 0$, then $y \geq 0$ and $\psi_{s} \geq 0$.

Proof. Let $\psi \in E^{*}$ be fixed. As observed in Section 4 , $\psi$ may be written in the form

$$
\psi=\psi^{(1)}-\psi^{(2)}+i\left(\psi^{(3)}-\psi^{(4)}\right),
$$

where $0 \leq \psi^{(j)} \in E^{*}$ for $1 \leq j \leq 4$. By Proposition [5.6] each $\psi^{(j)}$ has a decomposition

$$
\psi^{(j)}=\psi_{n}^{(j)}+\psi_{s}^{(j)},
$$

where $0 \leq \psi_{s}^{(j)} \in E_{s}^{*}$ and $0 \leq \psi_{n}^{(j)} \in E_{n}^{*}$ is given by $\psi_{n}^{(j)}(x)=\tau\left(x a_{j}\right), x \in E$, with $0 \leq a_{j} \in E^{\times}(1 \leq j \leq 4)$. Defining

$$
\psi_{n}=\psi_{n}^{(1)}-\psi_{n}^{(2)}+i\left(\psi_{n}^{(3)}-\psi_{n}^{(4)}\right)
$$

and

$$
\psi_{s}=\psi_{s}^{(1)}-\psi_{s}^{(2)}+i\left(\psi_{s}^{(3)}-\psi_{s}^{(4)}\right),
$$

it is clear that $\psi=\psi_{n}+\psi_{s}$ is the desired decomposition of $\psi$. Since $E_{n}^{*} \cap E_{s}^{*}=\{0\}$, it is clear that this decomposition is unique. The final statements of the theorem have already been observed in Proposition 5.6.

The following result, which has been obtained in [10], Theorem 5.11 for strongly symmetric spaces, is now a simple consequence of Theorem 5.8 .

Theorem 5.9. Let $E \subseteq S(\tau)$ be a strongly symmetrically normed space. For a functional $\psi \in E^{*}$ the following statements are equivalent:

(i) $\psi$ is normal;

(ii) there exists a unique element $y \in E^{\times}$such that

$$
\psi(x)=\tau(x y), \quad x \in E .
$$

If any of the preceding equivalent assertions are valid, then $\psi$ is hermitian if and only if $y=y^{*}$ and $\psi$ is positive if and only if $y \geq 0$.

Proof. It has already been observed in Section 4 that (ii) implies (i). Therefore, it is sufficient to show that (i) implies (ii). If $\psi$ is normal, then, by Theorem 5.8, there exist $y \in E^{\times}$and $\psi_{s} \in E_{s}^{*}$ such that $\psi(x)=\tau(x y)+\psi_{s}(x), x \in E$, which implies that $\psi_{s} \in E_{n}^{*}$. Since $E_{n}^{*} \cap E_{s}^{*}=\{0\}$, it follows that $\psi_{s}=0$. Consequently, $\psi(x)=\tau(x y), x \in E$. This suffices to prove the theorem.

Suppose that $E \subseteq S(\tau)$ is strongly symmetrically normed space. It follows from Theorem 5.8 that $E^{*}=E_{n}^{*} \oplus E_{s}^{*}$, and by Theorem 5.9 the space $E_{n}^{*}$ may be identified with the Köthe dual $E^{\times}$of $E$ via trace duality. For $\psi \in E^{*}$, the decomposition

$$
\psi=\psi_{n}+\psi_{s}, \quad \psi_{n} \in E_{n}^{*}, \psi_{s} \in E_{s}^{*},
$$

will be referred to as the Yosida-Hewitt decomposition. Define $P_{n}: E^{*} \rightarrow E^{*}$ by setting $P_{n} \psi=\psi_{n}, \psi \in E^{*}$, that is, $P_{n}$ is the linear projection onto $E_{n}^{*}$ along $E_{s}^{*}$, and let $P_{s}=I-P_{n}$. As follows from Proposition 5.6, both projections $P_{n}$ and $P_{s}$ 
are positive, that is, $P_{n} \psi \geq 0$ and $P_{s} \psi \geq 0$ whenever $0 \leq \psi \in E^{*}$. Consequently, $0 \leq P_{n} \psi \leq \psi$ and $0 \leq P_{s} \psi \leq \psi$ for all $0 \leq \psi \in E^{*}$. In particular, it follows from Corollary 4.3 that

$$
\left\|P_{n} \psi\right\|_{E^{*}} \leq\|\psi\|_{E^{*}} \quad \text { and } \quad\left\|P_{s} \psi\right\|_{E^{*}} \leq\|\psi\|_{E^{*}}, \quad 0 \leq \psi \in E^{*} .
$$

Every $\psi \in E^{*}$ may be written as

$$
\psi=\psi_{1}-\psi_{2}+i\left(\psi_{3}-\psi_{4}\right),
$$

where $0 \leq \psi_{j} \in E^{*}$ and $\left\|\psi_{1}\right\|_{E^{*}}+\left\|\psi_{2}\right\|_{E^{*}} \leq 2\|\psi\|_{E^{*}},\left\|\psi_{3}\right\|_{E^{*}}+\left\|\psi_{4}\right\|_{E^{*}} \leq 2\|\psi\|_{E^{*}}$ (see (4.1) in Section (4). This implies that the projections $P_{n}$ and $P_{s}$ are bounded with $\left\|P_{n}\right\|_{\mathcal{L}\left(E^{*}\right)} \leq 4$ and $\left\|P_{s}\right\|_{\mathcal{L}\left(E^{*}\right)} \leq 4$. In particular, $E_{n}^{*}$ and $E_{s}^{*}$ are normed closed subspaces of $E^{*}$.

Identifying $E_{n}^{*}$ with $E^{\times}$, it follows that $E_{n}^{*}$ is positively generated. It should be observed that the same holds for $E_{s}^{*}$. Indeed, it has already been observed in Section 4 that $\operatorname{Re} \psi, \operatorname{Im} \psi \in E_{s}^{*}$ whenever $\psi \in E_{s}^{*}$. If $\psi \in E_{s}^{*}$ is hermitian, then $\psi=\psi_{1}-\psi_{2}$ with $0 \leq \psi_{j} \in E^{*}, j=1,2$, Hence, $\psi=P_{s} \psi=P_{s} \psi_{1}-P_{s} \psi_{2}$, and it has been observed above that $P_{s} \psi_{j} \geq 0(j=1,2)$. This shows that $E_{s}^{*}$ is positively generated.

Corollary 5.10. Let $E \subseteq S(\tau)$ be a strongly symmetrically normed space. Then:

(i) The space $E_{n}^{*}$ is positively generated, and $w \psi, \psi w \in E_{n}^{*}$ whenever $\psi \in E_{n}^{*}$ and $w \in \mathcal{M}$.

(ii) The space $E_{s}^{*}$ is positively generated, and $w \psi, \psi w \in E_{s}^{*}$ whenever $\psi \in E_{s}^{*}$ and $w \in \mathcal{M}$.

Proof. (i) This follows immediately from the identification of $E_{n}^{*}$ with $E^{\times}$via trace duality.

(ii) That $E_{s}^{*}$ is positively generated has already been observed above. Therefore, to show that $\psi w \in E_{s}^{*}$ whenever $\psi \in E_{s}^{*}$ and $w \in \mathcal{M}$, we may assume that $0 \leq \psi \in E_{s}^{*}$. Let $J \subseteq E$ be an order dense order ideal on which $\psi$ vanishes. Since $|x| \in J$ whenever $x \in J$, it follows from Lemma 4.2 that $\psi w$ also vanishes on $J$, and so $\psi w \in E_{s}^{*}$. Since $w \psi=\bar{\psi} w^{*}$ and $\phi \in E_{s}^{*}$ if and only if $\bar{\phi} \in E_{s}^{*}$, it is now also clear that $w \psi \in E_{s}^{*}$ for all $\psi \in E_{s}^{*}$ and $w \in \mathcal{M}$.

Another consequence of the above results is the following proposition.

Proposition 5.11. Let $E \subseteq S(\tau)$ be a strongly symmetrically normed space. If $\psi \in E^{*}$, then the following statements are equivalent:

(i) $\psi$ is normal;

(ii) $\psi$ is completely additive;

(iii) $\psi\left(x e_{\alpha}\right) \rightarrow_{\alpha} 0$ and $\psi\left(e_{\alpha} x\right) \rightarrow_{\alpha} 0$ whenever $\left\{e_{\alpha}\right\}$ is a downward directed net in $P(\mathcal{M})$ such that $e_{\alpha} \downarrow_{\alpha} 0$.

Proof. That (i) implies (iii) follows immediately from Theorem 5.9 in combination with Lemma 4.11. It is easy to see that (iii) implies (ii), and so it remains to show that (ii) implies (i). Observe that it suffices to show that any completely additive singular functional is equal to zero, as follows from Theorem 5.8 and the fact, just observed, that any normal functional is completely additive.

For this purpose, suppose that $\psi \in E_{s}^{*}$ is completely additive. Given $e \in P_{f}(\mathcal{M})$, define $\psi^{e} \in \mathcal{M}^{*}$ by $\psi^{e}(z)=\psi(e z), z \in \mathcal{M}$. Since $\psi$ is completely additive, it is clear that $\psi^{e}$ is completely additive on projections, and so $\psi^{e} \in \mathcal{M}_{n}^{*}$ (see Theorem 
4.13). On the other hand, by Corollary 5.10 (ii), the functional $\psi e$ is singular on $E$, and hence vanishes on some order dense order ideal $J \subseteq E$. Therefore, the functional $\psi^{e}$ vanishes on $J \cap \mathcal{M}$, which is an order dense order ideal in $\mathcal{M}$ (cf. Remark 4.9). Hence, $\psi^{e} \in \mathcal{M}_{s}^{*}$, and so $\psi^{e}=0$.

Given $x \in E$, let $\left\{e_{\alpha}\right\}$ be a mutually orthogonal system in $P_{f}(\mathcal{M})$ such that $\sum_{\alpha} e_{\alpha}=\mathbf{1}$ and $e_{\alpha} x \in \mathcal{M}$ (since $\tau$ is semi-finite, such a system always exists). Now it follows from the complete additivity of $\psi$ that

$$
\psi(x)=\sum_{\alpha} \psi\left(e_{\alpha} x\right)=\sum_{\alpha} \psi^{e_{\alpha}}\left(e_{\alpha} x\right)=0 .
$$

Therefore, we may conclude that $\psi=0$, which completes the proof.

We end this section by proving another important property of the projections $P_{n}$ and $P_{s}$. In the proof, the following observation will be used.

Lemma 5.12. If $\left\{\psi_{n}\right\}_{n=1}^{\infty}$ is a sequence in $E_{s}^{*}$, then there exists an order dense order ideal $J \subseteq E$ on which all functionals $\psi_{n}$ vanish.

Proof. Since $E_{s}^{*}$ is positively generated (see Corollary [5.10), it may be assumed, without loss of generality, that $\psi_{n} \geq 0$ and that $\left\|\psi_{n}\right\|_{E^{*}} \leq 1$ for all $n$. Define $0 \leq \phi \in E^{*}$ by setting

$$
\phi=\sum_{n=1}^{\infty} 2^{-n} \psi_{n}
$$

Since $E_{s}^{*}$ is closed, it follows that $0 \leq \phi \in E_{s}^{*}$, and so $\phi$ vanishes on some order dense order ideal $J \subseteq E$. If $x \in J^{+}$, then $0 \leq \psi_{n}(x) \leq 2^{n} \phi(x)=0$, which implies that $\psi_{n}(x)=0$. Hence, all functionals $\psi_{n}, n \in \mathbb{N}$, vanish on $J$.

Theorem 5.13. If $E \subseteq S(\tau)$ is a strongly symmetrically normed space, then the projections $P_{n}$ and $P_{s}$ are sequentially $\sigma\left(E^{*}, E\right)$-continuous.

Proof. Let $\left\{\psi_{k}\right\}_{k=1}^{\infty}$ be a sequence in $E^{*}$ such that $\psi_{k} \stackrel{\sigma\left(E^{*}, E\right)}{\rightarrow} 0$ as $k \rightarrow \infty$. It suffices to show that $P_{n} \psi_{k} \stackrel{\sigma\left(E^{*}, E\right)}{\rightarrow} 0$ as $k \rightarrow \infty$. It follows from Lemma 5.12 that there exists an order dense order ideal $J \subseteq E$ on which all functionals $P_{s} \psi_{k}$ vanish. Let $0 \leq x \in E$ be fixed. By Lemma 4.8, there exists a family $\left\{x_{\alpha}: \alpha \in \mathbb{A}\right\}$ in $J^{+}$ such that $\sum_{\alpha \in \mathbb{A}} x_{\alpha}=x$. For $k \in \mathbb{N}$ define the finitely additive set function $\nu_{k}$ on the $\sigma$-algebra $2^{\mathbb{A}}$ of all subsets of the index set $\mathbb{A}$ by setting

$$
\nu_{k}(A)=\psi_{k}\left(\sum_{\alpha \in A} x_{\alpha}\right), \quad A \in 2^{\mathbb{A}} .
$$

Since

$$
\sup \left\{\left|\nu_{k}(A)\right|: A \in 2^{\mathbb{A}}\right\} \leq\left\|\psi_{k}\right\|_{E^{*}}\|x\|_{E},
$$

each $\nu_{k}$ is of bounded variation. Furthermore, since $\psi_{k} \rightarrow 0$ for the $\sigma\left(E^{*}, E\right)$ topology, it is clear that

$$
\lim _{k \rightarrow \infty} \nu_{k}(A)=\lim _{k \rightarrow \infty} \psi_{k}\left(\sum_{\alpha \in A} x_{\alpha}\right)=0, \quad A \in 2^{\mathbb{A}} .
$$

Consequently, it follows from Phillip's lemma (see 4], Chapter VII, or [26], Theorem III.1.28) that

$$
\lim _{k \rightarrow \infty} \sum_{\alpha \in \mathbb{A}}\left|\psi_{k}\left(x_{\alpha}\right)\right|=\lim _{k \rightarrow \infty} \sum_{\alpha \in \mathbb{A}}\left|\nu_{k}(\{\alpha\})\right|=0
$$


Since $\left(P_{s} \psi_{k}\right)\left(x_{\alpha}\right)=0$ for all $k \in \mathbb{N}$ and all $\alpha \in \mathbb{A}$, this shows that

$$
\lim _{k \rightarrow \infty} \sum_{\alpha \in \mathbb{A}}\left|\left(P_{n} \psi_{k}\right)\left(x_{\alpha}\right)\right|=0 .
$$

Using the fact that $P_{n} \psi_{k}$ is normal, it follows that

$$
\begin{aligned}
\lim _{k \rightarrow \infty}\left(P_{n} \psi_{k}\right)(x) & =\lim _{k \rightarrow \infty}\left(P_{n} \psi_{k}\right)\left(\sum_{\alpha \in \mathbb{A}} x_{\alpha}\right) \\
& =\lim _{k \rightarrow \infty} \sum_{\alpha \in \mathbb{A}}\left(P_{n} \psi_{k}\right)\left(x_{\alpha}\right)=0,
\end{aligned}
$$

and this suffices to complete the proof.

Corollary 5.14. If $E \subseteq S(\tau)$ is a strongly symmetrically normed space, then the spaces $E_{n}^{*}$ and $E_{s}^{*}$ are sequentially $\sigma\left(E^{*}, E\right)$-complete. Consequently, the Köthe dual $E^{\times}$is sequentially $\sigma\left(E^{\times}, E\right)$-complete.

Proof. Since $E^{*}$ is sequentially $\sigma\left(E^{*}, E\right)$-complete, the first statement follows immediately from Theorem [5.13. The second statement now follows via Theorem 5.9 .

Recall that a strongly symmetric space $E \subseteq S(\tau)$ is called a $K B$-space if every increasing sequence $\left\{x_{n}\right\}_{n=1}^{\infty} \subseteq E^{+}$satisfying $\sup _{n}\left\|x_{n}\right\|_{E}<\infty$ is convergent in $E$. It is not difficult to show that $E$ is a $K B$-space if and only if $E$ has order continuous norm and the Fatou property. We use the above result to present a simple alternative proof of the following characterization of $K B$-spaces, which has been obtained in $[9]$.

Theorem 5.15. If $E \subseteq S(\tau)$ is a strongly symmetric space, then the following statements are equivalent:

(i) $E$ is a $K B$-space;

(ii) $E$ is weakly sequentially complete.

Proof. Using the fact that increasing weakly convergent sequences in $E^{+}$are norm convergent (see e.g. the proof of implication (i) $\Rightarrow$ (ii) of [9], Proposition 3.2), it is easy to see that (ii) implies (i). To prove the converse implication, suppose that $E$ is a $K B$-space. Applying Corollary $\left[5.14\right.$ to the space $E^{\times}$, it follows that $E^{\times \times}$ is sequentially $\sigma\left(E^{\times \times}, E^{\times}\right)$-complete. Since $E$ has the Fatou property, it follows from [10, Proposition 5.14, that $E=E^{\times \times}$. Furthermore, the norm in $E$ is order continuous, and so, by Proposition 4.12 and Theorem 5.9, $E^{*}$ may be identified with the Köthe dual $E^{\times}$via trace duality. Consequently, $E$ is sequentially $\sigma\left(E, E^{*}\right)$ complete.

We conclude this section with some remarks concerning the Yosida-Hewitt projections $P_{n}, P_{s}$. As noted following Theorem 5.9 above, the projections $P_{n}, P_{s}$ are bounded positive projections which are contractive on the positive cone of $E$ in the sense that $0 \leq P_{n}, P_{s} \leq I$. It seems to be an interesting question as to whether these projections are contractive on $E$, that is, are projections of norm 1 . This is certainly the case if $\mathcal{M}$ is commutative and in the case of trace ideals, as shown in [12. We note further that it was shown by Dixmier [5] that the decomposition of the Banach dual of $B(H)$ into normal and singular functionals is an $l^{1}$-decomposition, and the same result was proved subsequently by Takesaki 24 for more general von 
Neumann algebras. If follows, of course, that in each of these cases the YosidaHewitt projections are contractive. However, in the general setting considered in this paper, this question remains open.

\section{Some Consequences}

In this section we will discuss some consequences of the non-commutative YosidaHewitt decomposition (Theorem [5.8). As before, $\mathcal{M} \subseteq B(H)$ is a semi-finite von Neumann algebra, equipped with a fixed semi-finite, normal and faithful trace $\tau$, and $E \subseteq S(\tau)$ is a strongly symmetrically normed space (as always, satisfying $\left.c_{E}=\mathbf{1}\right)$. Some further notation and terminology is needed.

Definition 6.1. (i) The subset $E^{o c} \subseteq E$ is defined by setting

$$
E^{o c}=\left\{x \in E:|x| \geq x_{\alpha} \downarrow_{\alpha} 0 \Rightarrow\left\|x_{\alpha}\right\|_{E} \downarrow_{\alpha} 0\right\},
$$

and the elements of $E^{o c}$ are called elements of order continuous norm.

It is not difficult to show that $E^{o c}$ is also given by

$$
E^{o c}=\left\{x \in E:|x| \geq x_{n} \downarrow_{n} 0 \Rightarrow\left\|x_{n}\right\|_{E} \downarrow 0 \text { as } n \rightarrow \infty\right\} .
$$

Evidently, $E$ has order continuous norm if and only if $E^{o c}=E$. Observe furthermore that $E^{o c} \subseteq E^{b}\left(=\overline{\mathcal{F}}(\tau)^{E}\right)$. Indeed, given $x \in E^{o c}$, there exists an upwards directed net $\left\{x_{\alpha}\right\} \subseteq \mathcal{F}(\tau)^{+}$such that $0 \leq x_{\alpha} \uparrow_{\alpha}|x|$, that is, $|x| \geq|x|-x_{\alpha} \downarrow_{\alpha} 0$. Hence, ||$|x|-x_{\alpha} \|_{E} \downarrow_{\alpha} 0$, and so $|x| \in E^{b}$, which implies that $x \in E^{b}$.

A useful alternative characterization of $E^{o c}$ will be given in the next proposition. The following notation will be used. If $A \subseteq E$ and $B \subseteq E^{*}$, then we set

$$
A^{\perp}=\left\{\phi \in E^{*}: \phi(x)=0 \forall x \in A\right\},{ }^{\perp} B=\{x \in E: \phi(x)=0 \forall \phi \in B\},
$$

which are closed linear subspaces of $E^{*}$ and $E$, respectively.

Proposition 6.2. The set $E^{o c}$ of all elements of order continuous norm in $E$ is also given by $E^{o c}={ }^{\perp} E_{s}^{*}$.

Proof. Suppose that $x \in E^{o c}$ and $0 \leq \psi \in E_{s}^{*}$. Let $J \subseteq E$ be an order dense order ideal on which $\psi$ vanishes. By Lemma 4.8, there exists an upwards directed system $\left\{x_{\alpha}\right\} \subseteq J^{+}$such that $0 \leq x_{\alpha} \uparrow_{\alpha}|x|$, which implies that $\left\||x|-x_{\alpha}\right\|_{E} \rightarrow_{\alpha} 0$. Hence, $\psi\left(x_{\alpha}\right) \rightarrow_{\alpha} \psi(|x|)$, and so $\psi(|x|)=0$. It now follows from Lemma 4.2 (see (4.4)) that $\psi(x)=0$. Since $E_{s}^{*}$ is positively generated (see Corollary [5.10), this suffices to show that $E^{o c} \subseteq^{\perp} E_{s}^{*}$.

For the proof of the reverse inclusion, suppose that $x \in E$ and $\psi(x)=0$ for all $x \in E_{s}^{*}$. If $x=v|x|$ is the polar decomposition of $x$, then it follows from Corollary 5.10 that $\psi(|x|)=(\psi v)(x)=0$. Suppose now that $|x| \geq x_{\alpha} \downarrow_{\alpha} 0$ and that $0 \leq \phi \in E^{*}$. Let $\phi=\phi_{n}+\phi_{s}$ be the Yosida-Hewitt decomposition of $\phi$, where $0 \leq \phi_{n} \in E_{n}^{*}$ and $0 \leq \phi_{s} \in E_{s}^{*}$ (see Theorem [5.8). Since $0 \leq \phi_{s}\left(x_{\alpha}\right) \leq \phi_{s}(|x|)=0$, it follows that $\phi_{s}\left(x_{\alpha}\right)=0$ for all $\alpha$. Hence, $\phi\left(x_{\alpha}\right)=\phi_{n}\left(x_{\alpha}\right) \downarrow_{\alpha} 0$. Since $E^{*}$ is positively generated, this implies that $\phi\left(x_{\alpha}\right) \rightarrow 0$ for all $\phi \in E^{*}$, and so $\left\|x_{\alpha}\right\|_{E} \downarrow_{\alpha} 0$. Therefore $x \in E^{o c}$, which shows that ${ }^{\perp} E_{s}^{*} \subseteq E^{o c}$. The proof is complete.

Corollary 6.3. The set $E^{o c}$ is a closed linear subspace of $E$ with the property that $u x v \in E^{o c}$ whenever $x \in E^{o c}$ and $u, v \in \mathcal{M}$. In particular, $E^{o c}$ is an order ideal in E. 
Proof. From Proposition 6.2 it is clear that $E^{o c}$ is a closed linear subspace of $E$. Suppose that $x \in E^{o c}$ and $u, v \in \mathcal{M}$. If $\phi \in E_{s}^{*}$, then it follows from Corollary 5.10 that $v \phi u \in E_{s}^{*}$, and so, by Proposition 6.2. $\phi(u x v)=(v \phi u)(x)=0$. Using the same proposition once more, it follows that $u x v \in E^{o c}$.

Evidently, $|x| \in E^{o c}$ whenever $x \in E^{o c}$, and if $0 \leq x \leq y$ with $y \in E^{o c}$ and $x \in E$, then $x \in E^{o c}$. If $x \in E^{o c}$ with polar decomposition $x=w|x|$, then $x^{*}=w^{*} x w^{*}$, and so, $x^{*} \in E^{o c}$. Hence, $E^{o c}$ is an order ideal.

It may happen that $E^{o c}=\{0\}$. Indeed, this the case, for example, if $E=\mathcal{M}=$ $L_{\infty}(\nu)$, where $\nu$ is an atomless measure. The following proposition presents several characterizations as to when $E^{o c}$ is order dense in $E$.

Proposition 6.4. If $E \subseteq S(\tau)$ is a strongly symmetrically normed space, then the following statements are equivalent:

(i) $\left(E^{o c}\right)^{\perp}=E_{s}^{*}$;

(ii) $E_{s}^{*}$ is $\sigma\left(E^{*}, E\right)$-closed in $E^{*}$;

(iii) $E^{o c}$ separates the points of $E_{n}^{*}$;

(iv) $E^{o c}$ is order dense in $E$;

(v) the map $\left.\phi \longmapsto \phi\right|_{E^{o c}}, \phi \in E_{n}^{*}$, is a linear isometry from $E_{n}^{*}$ onto $\left(E^{o c}\right)^{*}$.

Proof. (i) $\Leftrightarrow$ (ii). It follows from Proposition 6.2 that

$$
\left(E^{o c}\right)^{\perp}=\left({ }^{\perp} E_{s}^{*}\right)^{\perp}={\overline{E_{s}^{*}}}^{\sigma\left(E^{*}, E\right)},
$$

and so statements (i) and (ii) are equivalent.

(i) $\Leftrightarrow$ (iii). Observe first that statement (iii) is equivalent to saying that $E_{n}^{*} \cap$ $\left(E^{o c}\right)^{\perp}=\{0\}$. If (i) holds, then $E_{n}^{*} \cap\left(E^{o c}\right)^{\perp}=E_{n}^{*} \cap E_{s}^{*}=\{0\}$. Suppose now that (iii) holds. Given $\phi \in\left(E^{o c}\right)^{\perp}$, let $\phi=\phi_{n}+\phi_{s}$ be its Yosida-Hewitt decomposition. By Proposition 6.2, $\phi_{s} \in\left(E^{o c}\right)^{\perp}$, and so $\phi_{n} \in\left(E^{o c}\right)^{\perp} \cap E_{n}^{*}=\{0\}$. Therefore, $\phi \in E_{s}^{*}$. This shows that $\left(E^{o c}\right)^{\perp} \subseteq E_{s}^{*}$. Since the reverse inclusion is always valid (see (6.2) ), statement (i) follows.

(iii) $\Rightarrow$ (iv). Setting $P\left(E^{o c}\right)=P(\mathcal{M}) \cap E^{o c}$, define the projection $c \in P(\mathcal{M})$ by setting $c=\bigvee P\left(E^{o c}\right)$. We claim that $s(x) \leq c$ (and hence, $x=x c$ ) for all $x \in E^{o c}$. Indeed, if $x \in E^{o c}$, then $s e^{|x|}(s, \infty) \leq|x| e^{|x|}(s, \infty) \in E^{o c}$, and so $e^{|x|}(s, \infty) \in E^{o c}$ for all $s>0$. Since $s(x)=s(|x|)=e^{|x|}(0, \infty)=\bigvee_{s>0} e^{|x|}(s, \infty)$, our claim follows.

It will be shown next that $c=1$. Suppose that $c<\mathbf{1}$ and let $p \in P_{f}(\mathcal{M})$ be such that $0<p \leq c^{\perp}$. Defining $\phi \in E_{n}^{*}$ by setting $\phi(x)=\tau(x p), x \in E$, it follows that $\phi(x)=\phi(x c)=\tau(x c p)=0$ for all $x \in E^{o c}$. By (iii), this implies that $\phi=0$, which is a contradiction, as $\phi(p)=\tau(p)>0$. Therefore, $c=\mathbf{1}$.

Let $0<p \in P(E)$ be given. If $p e=0$ for all $e \in P\left(E^{o c}\right)$, then $e \leq p^{\perp}$ for all $e \in P\left(E^{o c}\right)$, and so $p^{\perp}=\mathbf{1}$, that is, $p=0$. This is a contradiction, and hence there exists $e \in P\left(E^{o c}\right)$ such that $p e \neq 0$. Defining $q=r(p e)$, it is clear that $0<q \leq p$. Furthermore, $q \sim s(p e)$, and so there exists a partial isometry $v \in \mathcal{M}$ such that $q=v^{*} s(p e) v$. Since $s(p e) \leq e$, it is clear that $s(p e) \in P\left(E^{o c}\right)$, and hence it follows from Corollary 6.3 that $q \in P\left(E^{o c}\right)$. Consequently, $E^{o c}$ satisfies condition (iv) of Lemma 4.8, and so we may conclude that $E^{o c}$ is order dense.

(iv) $\Rightarrow$ (v). If $\phi \in E_{n}^{*}$, then it follows from Theorem 5.9 that there exists $y \in E^{\times}$ such that $\phi(x)=\tau(x y), x \in E$. Since $E^{o c}$ is order dense in $E$, it follows from Lemma 4.8 (iii) in combination with the argument used in Remark 3.6(i) (replacing $\mathcal{F}(\tau)$ by $\left.E^{o c}\right)$ that $\|\phi\|_{E^{*}}=\left\|\left.\phi\right|_{E^{o c}}\right\|_{\left(E^{o c}\right)^{*}}$. This shows that the map $\left.\phi \longmapsto \phi\right|_{E^{o c}}$, 
$\phi \in E_{n}^{*}$, is a linear isometry from $E_{n}^{*}$ into $\left(E^{o c}\right)^{*}$. It remains to be shown that this map is onto. Given $\psi \in\left(E^{o c}\right)^{*}$, it follows from the Hahn-Banach theorem that there exists $\phi \in E^{*}$ such that $\left.\phi\right|_{E^{o c}}=\psi$. If $\phi=\phi_{n}+\phi_{s}$ is the Yosida-Hewitt decomposition of $\phi$, then it follows from Proposition 6.2 that $\left.\phi_{s}\right|_{E^{o c}}=0$, and so $\psi=\left.\phi_{n}\right|_{E^{o c}}$. This shows that (v) holds.

Evidently, (v) implies (iii), and therefore the proof is complete.

In the case of Banach function spaces the above results may be found in [30, Section 72. Similar results in the setting of Banach lattices may be found in [31, Sections 102 and 103.

If the von Neumann algebra $\mathcal{M}$ is either atomless (that is, does not contain any minimal projections) or $\mathcal{M}$ is atomic with all minimal projections having equal trace, then the above results may be improved. This will be shown in the following propositions.

Proposition 6.5. Let $\mathcal{M}$ be an atomless von Neumann algebra. If $E \subseteq S(\tau)$ is a strongly symmetrically normed space, then the following conditions are equivalent:

(i) $E^{o c} \neq\{0\}$;

(ii) for every $\varepsilon>0$ there exists $\delta>0$ such that $\|p\|_{E} \leq \varepsilon$ whenever $p \in P(E)$ satisfies $\tau(p) \leq \delta$;

(iii) $E^{o c}=E^{b}$;

(iv) $E^{o c}$ is order dense in $E$.

Proof. (i) $\Rightarrow$ (ii). Since $E^{o c} \neq\{0\}$ there exists a projection $e \in P\left(E^{o c}\right)$ satisfying $0<\tau(e)<\infty$. Furthermore, $\mathcal{M}$ is assumed to be atomless, and so there exists a sequence $\left\{e_{n}\right\}_{n=1}^{\infty}$ in $P(\mathcal{M})$ such that $e \geq e_{n} \downarrow_{n} 0$ and $\tau\left(e_{n}\right)=2^{-n}$ for all $n$. Since $e \in E^{o c}$, this implies that $\left\|e_{n}\right\|_{E} \downarrow 0$ as $n \rightarrow \infty$. Given $\varepsilon>0$, there exists $n_{0} \in \mathbb{N}$ such that $\left\|e_{n_{0}}\right\|_{E} \leq \varepsilon$. Let $\delta=\tau\left(e_{n_{0}}\right)$. If $p \in P(E)$ and $\tau(p) \leq \delta$, then $\mu(p) \leq \chi_{[0, \delta)}=\mu\left(e_{n_{0}}\right)$, and hence $\|p\|_{E} \leq\left\|e_{n_{0}}\right\|_{E} \leq \varepsilon$.

(ii) $\Rightarrow$ (iii). As observed before, the inclusion $E^{o c} \subseteq E^{b}$ always holds. Therefore, it is sufficient to show that $\mathcal{F}(\tau) \subseteq E^{o c}$, that is, $q \in P\left(E^{o c}\right)$ whenever $q \in P(E)$ and $\tau(q)<\infty$. Fixing $q \in P(E)$ with $\tau(q)<\infty$, let $\left\{a_{\beta}\right\}$ be a decreasing net in $E^{+}$such that $q \geq a_{\beta} \downarrow_{\beta} 0$. Given $\varepsilon>0$, let $\delta>0$ be such that $\|p\|_{E} \leq \varepsilon$ for all $p \in P(E)$ satisfying $\tau(p) \leq \delta$. Since $\tau(q)<\infty$, the normality of the trace implies that $\tau\left(a_{\beta}\right) \downarrow_{\beta} 0$. Observing that $0 \leq \varepsilon e^{a_{\beta}}(\varepsilon, \infty) \leq a_{\beta}$, it follows that $\tau\left(e^{a_{\beta}}(\varepsilon, \infty)\right) \rightarrow_{\beta} 0$, and so there exists $\beta_{0}$ such that $\tau\left(e^{a_{\beta}}(\varepsilon, \infty)\right) \leq \delta$ whenever $\beta \geq \beta_{0}$. It follows from $0 \leq a_{\beta} \leq q$ that

$$
a_{\beta}=a_{\beta} e^{a_{\beta}}(\varepsilon, \infty)+a_{\beta} e^{a_{\beta}}[0, \varepsilon] \leq e^{a_{\beta}}(\varepsilon, \infty)+\varepsilon q,
$$

and hence $\left\|a_{\beta}\right\|_{E} \leq \varepsilon\left(1+\|q\|_{E}\right)$ whenever $\beta \geq \beta_{0}$. This shows that $q \in E^{o c}$, which is sufficient to establish assertion (iii).

Evidently, (iii) implies (iv) and (iv) implies (i). Therefore, the proof is complete.

Proposition 6.6. Suppose that the von Neumann algebra $\mathcal{M}$ is atomic and that all minimal projections have the same trace. If $E \subseteq S(\tau)=\mathcal{M}$ is a strongly symmetrically normed space, then $E^{o c}=E^{b}$, which is equal to the closed linear span of all minimal projections.

Proof. First observe that it follows from the assumption on $\mathcal{M}$ that $\mathcal{F}(\tau)$ is the linear span of all minimal projections. Therefore, $E^{b}$ is equal to the closed linear 
span of all minimal projections. Furthermore, it is clear that $E^{o c}$ contains all minimal projections (indeed, if $p \in P(\mathcal{M})$ is minimal, then $p \mathcal{M} p=\mathbb{C} p$, and so, if $p \geq x_{\alpha} \downarrow_{\alpha} 0$, then $x_{\alpha}=\lambda_{\alpha} p$ with $\lambda_{\alpha} \downarrow_{\alpha} 0$ in $\left.\mathbb{R}\right)$. Consequently, $\mathcal{F}(\tau) \subseteq E^{o c}$, and this suffices to show that $E^{o c}=E^{b}$. The proof is complete.

The following corollary has essentially been obtained in 12, Proposition 2.7.

Corollary 6.7. Suppose that the von Neumann algebra $\mathcal{M}$ is atomic and that all minimal projections have the same trace. If $E \subseteq \mathcal{M}$ is a strongly symmetrically normed space, then a functional $\psi \in E^{*}$ is singular if and only if $\psi$ vanishes on all minimal projections. Furthermore, defining for each $y \in E^{\times}$the linear functional $\phi_{y}$ on $E^{b}$ by setting

$$
\phi_{y}(x)=\tau(x y), \quad x \in E^{b},
$$

the map $\phi \longmapsto \phi_{y}$ is a linear isometry from $E^{\times}$onto $\left(E^{b}\right)^{*}$.

Proof. By Proposition 6.6. $E^{o c}=E^{b}$, which clearly implies that $E^{o c}$ is order dense in $E$. Therefore, condition (iv) of Proposition 6.4 is satisfied. The first statement of the present corollary is now an immediate consequence of part (i) of Proposition 6.4, and the second statement is a simple reformulation of part (v) of the same proposition.

Some important alternative descriptions of elements of order continuous norm will be discussed next. For this purpose, the following notions are introduced. As before, $E$ is assumed to be a strongly symmetrically normed space of $\tau$-measurable operators (always satisfying $c_{E}=\mathbf{1}$ ).

Definition 6.8. (i) An element $x \in E$ is said to have absolutely continuous norm if $\left\|e_{n} x e_{n}\right\|_{E} \rightarrow 0$ for every sequence $\left\{e_{n}\right\}_{n=1}^{\infty}$ in $P(\mathcal{M})$ satisfying $e_{n} \downarrow_{n} 0$. The set of all elements of absolutely continuous norm is denoted by $E^{a n}$.

(ii) A subset $\mathcal{A}$ of $E$ is said to be of uniformly absolutely continuous norm if

$$
\sup \left\{\left\|e_{n} x e_{n}\right\|_{E}: x \in \mathcal{A}\right\} \rightarrow 0
$$

as $n \rightarrow \infty$ for all sequences $\left\{e_{n}\right\}_{n=1}^{\infty}$ in $P(\mathcal{M})$ satisfying $e_{n} \downarrow 0$.

It is clear from the definition that $E^{a n}$ is a linear subspace of $E$ and that $x^{*} \in E^{a n}$ whenever $x \in E^{a n}$. It is also clear that any set of uniformly absolutely continuous norm is contained in $E^{a n}$. Furthermore, it should be observed that if $0 \leq y \in E^{a n}$, then the set

$$
[0, y]=\{x \in E: 0 \leq x \leq y\}
$$

is of uniformly absolutely continuous norm. In due course, it will be shown that actually $E^{a n}=E^{o c}$ (see Proposition 6.12). The following lemma collects together some of the elementary properties of the subspace $E^{a n}$.

Lemma 6.9. (i) $E^{o c} \subseteq E^{a n}$;

(ii) $E^{a n} \subseteq S_{0}(\tau)$;

(iii) if $x \in E^{a n}$, then $\left\|e_{n} x e_{n}\right\|_{E} \rightarrow 0$ whenever $\left\{e_{n}\right\}_{n=1}^{\infty}$ is a sequence in $P(\mathcal{M})$ satisfying $\tau\left(e_{n}\right) \rightarrow 0$ as $n \rightarrow \infty$. 
Proof. (i) It follows from Corollary 6.3 that each element of $E^{o c}$ is a linear combination of at most four positive elements. Therefore, it is sufficient to show that $\left(E^{o c}\right)^{+} \subseteq E^{a n}$. Suppose that $0 \leq x \in E^{o c}$ and that $e_{n} \downarrow_{n} 0$ in $P(\mathcal{M})$. Since

$$
\begin{aligned}
\mu\left(e_{n} x e_{n}\right) & =\mu\left(\left(x^{1 / 2} e_{n}\right)^{*}\left(x^{1 / 2} e_{n}\right)\right)=\mu\left(\left(x^{1 / 2} e_{n}\right)\left(x^{1 / 2} e_{n}\right)^{*}\right) \\
& =\mu\left(x^{1 / 2} e_{n} x^{1 / 2}\right)
\end{aligned}
$$

and $x \geq x^{1 / 2} e_{n} x^{1 / 2} \downarrow_{n} 0$ (see [10], Proposition 1.3), it follows that

$$
\left\|e_{n} x e_{n}\right\|_{E}=\left\|x^{1 / 2} e_{n} x^{1 / 2}\right\|_{E} \downarrow_{n} 0
$$

This shows that $x \in E^{a n}$.

(ii) To show that $x \in S_{0}(\tau)$ whenever $x \in E^{a n}$, it may be assumed, without loss of generality, that $x \in\left(E^{a n}\right)^{+}$. Suppose that $x \notin S_{0}(\tau)$, that is, there exists $s>0$ such that $\tau\left(e^{x}(s, \infty)\right)=\infty$. This implies that $\mu(x) \geq s \mathbf{1}$, and so $\mathcal{M} \subseteq E$. Since the trace is semi-finite, it follows that there exists an increasing sequence $\left\{p_{n}\right\}_{n=1}^{\infty}$ in $P(\mathcal{M})$ such that $p_{n} \leq e^{x}(s, \infty)$ and $n \leq \tau\left(p_{n}\right)<\infty$ for all $n$. Defining $p=\sup _{n} p_{n}$ and $e_{n}=p-p_{n}$ for all $n$, it is clear that $e_{n} \downarrow_{n} 0, \tau\left(e_{n}\right)=\infty$ and $e_{n} \leq e^{x}(s, \infty)$ for all $n$. Since $x \in E^{a n}$, it follows that $\left\|e_{n} x e_{n}\right\|_{E} \rightarrow 0$ as $n \rightarrow \infty$. On the other hand, $x e^{x}(s, \infty) \geq s e^{x}(s, \infty)$, and so

$$
e_{n} x e_{n}=e_{n} x e^{x}(s, \infty) e_{n} \geq s e_{n} e^{x}(s, \infty) e_{n}=s e_{n},
$$

which implies that $\left\|e_{n} x e_{n}\right\|_{E} \geq s\left\|e_{n}\right\|_{E}$ for all $n$. Since $\tau\left(e_{n}\right)=\infty$, it is also clear that $\mu\left(e_{n}\right)=\mu(\mathbf{1})$, and hence $\left\|e_{n}\right\|_{E}=\|\mathbf{1}\|_{E}$ for all $n$. Consequently, $\left\|e_{n} x e_{n}\right\|_{E} \geq$ $s\|\mathbf{1}\|_{E}$ for all $n$, which is clearly a contradiction. Therefore, it may be concluded that $x \in S_{0}(\tau)$, and this completes the proof of part (ii).

(iii) Suppose that $x \in E^{a n}$ and that $\left\{e_{n}\right\}_{n=1}^{\infty}$ is a sequence in $P(\mathcal{M})$ such that $\tau\left(e_{n}\right) \rightarrow 0$ but that $\left\|e_{n} x e_{n}\right\|_{E} \nrightarrow 0$. By passing to a subsequence, it may be assumed that $\tau\left(e_{n}\right) \leq 2^{-n}$ and $\left\|e_{n} x e_{n}\right\|_{E} \geq \delta$ for some $0<\delta \in \mathbb{R}$ and all $n$. Defining $p_{n} \in P(\mathcal{M})$ by setting

$$
p_{n}=\bigvee_{k=n}^{\infty} e_{k},
$$

it is clear that $p_{n} \downarrow_{n}$ and that $\tau\left(p_{n}\right) \leq \sum_{k=n}^{\infty} \tau\left(e_{k}\right) \leq 2^{-n+1}$. Consequently, $p_{n} \downarrow_{n} 0$, and so the hypothesis on $x$ implies that $\left\|p_{n} x p_{n}\right\|_{E} \rightarrow 0$ as $n \rightarrow \infty$. On the other hand,

$$
0<\delta \leq\left\|e_{n} x e_{n}\right\|_{E}=\left\|e_{n} p_{n} x p_{n} e_{n}\right\|_{E} \leq\left\|p_{n} x p_{n}\right\|_{E}
$$

for all $n$, which is absurd. This suffices for the proof of part (iii) of the lemma.

In the proof of the next proposition, the following observations will be used. A projection $p \in P(\mathcal{M})$ is called $\sigma$-finite (with respect to the trace $\tau$ ) if there exists a sequence $\left\{p_{n}\right\}_{n=1}^{\infty}$ in $P(\mathcal{M})$ such that $p_{n} \uparrow p$ and $\tau\left(p_{n}\right)<\infty$ for all $n$.

Lemma 6.10. (i) If $p \in P(\mathcal{M})$ and $\left\{p_{n}\right\}_{n=1}^{\infty}$ is a sequence of $\sigma$-finite projections in $P(\mathcal{M})$ such that $p=\sup _{n} p_{n}$, then $p$ is a $\sigma$-finite projection.

(ii) If $\left\{x_{n}\right\}_{n=1}^{\infty}$ is a sequence in $S_{0}(\tau)$, then there exists a $\sigma$-finite projection $p \in P(\mathcal{M})$ such that $x_{n}=p x_{n} p$ for all $n$. 
Proof. (i) For each $n \in \mathbb{N}$, let $p_{n k} \in P(\mathcal{M})$ be such that $p_{n k} \uparrow_{k} p_{n}$ and $\tau\left(p_{n k}\right)<\infty$ for all $k \in \mathbb{N}$. Defining $q_{n} \in P(\mathcal{M})$ by setting

$$
q_{n}=\sup \left\{p_{i j}: 1 \leq i \leq n, 1 \leq j \leq n\right\}, \quad n \in \mathbb{N},
$$

it is easy to see that $q_{n} \uparrow_{n} p$ and $\tau\left(q_{n}\right)<\infty$ for all $n$.

(ii) If $x \in S_{0}(\tau)$, then $\tau\left(e^{|x|}(1 / n, \infty)\right)<\infty$ for all $n \in \mathbb{N}$, and so the support projection $s(x)=s(|x|)=e^{|x|}(0, \infty)$ is $\sigma$-finite. Since $x^{*} \in S_{0}(\tau)$, also the range projection $r(x)=s\left(x^{*}\right)$ is $\sigma$-finite. Therefore, it follows from (i) that the projection $p=s(x) \vee r(x)$ is $\sigma$-finite, and it is clear that $x=p x p$. If $\left\{x_{n}\right\}_{n=1}^{\infty}$ is a sequence in $S_{0}(\tau)$, then it follows from the above observation, in combination with (i), that the projection $p=\sup _{n} s\left(x_{n}\right) \vee r\left(x_{n}\right)$ is $\sigma$-finite and satisfies $x_{n}=p x_{n} p$ for all $n$. The proof is complete.

For Banach function spaces the result of the following theorem goes back to [20, Chapter II, Section 2, Lemma 2. A non-commutative version of this result may be found in [3], under the additional assumptions that the von Neumann algebra $\mathcal{M}$ does not contain any minimal projections and that $E=E(\tau)$ (see (3.2) $)$, where $E(0, \infty)$ is a rearrangement invariant Banach function space with order continuous norm. A similar result is obtained in [22], where it is assumed that $E=E(\tau)$, where $E(0, \infty)$ is a rearrangement invariant Banach function space which is either minimal or maximal.

Theorem 6.11. If $\left\{x_{n}\right\}_{n=1}^{\infty}$ is a sequence in $E^{a n}$, then the following statements are equivalent:

(i) $\left\|x_{n}\right\|_{E} \rightarrow 0$ as $n \rightarrow \infty$;

(ii) $x_{n} \rightarrow 0$ with respect to the measure topology, and the set $\left\{x_{n}: n \in \mathbb{N}\right\}$ is of uniformly absolutely continuous norm.

Proof. (i) $\Rightarrow$ (ii). The embedding of $E$ into $S(\tau)$ is continuous with respect to the measure topology, and so $x_{n} \stackrel{\mathcal{T}_{m}}{\rightarrow} 0$ in $S(\tau)$. To see that $\left\{x_{n}\right\}_{n=1}^{\infty}$ has uniformly absolutely continuous norm, suppose that $\left\{e_{k}\right\}_{k=1}^{\infty}$ is a sequence in $P(\mathcal{M})$ satisfying $e_{k} \downarrow 0$ and let $\varepsilon>0$ be given. Choosing $N \in \mathbb{N}$ such that $\left\|x_{n}\right\|_{E} \leq \varepsilon$ for all $n \geq N$, it follows that

$$
\left\|e_{k} x_{n} e_{k}\right\|_{E} \leq\left\|x_{n}\right\|_{E} \leq \varepsilon, \quad n \geq N,
$$

for all $k \in \mathbb{N}$. Since the elements $\left\{x_{n}\right\}_{n=1}^{N-1}$ are assumed to have absolutely continuous norm, there exists $K \in \mathbb{N}$ such that $\left\|e_{k} x_{n} e_{k}\right\|_{E} \leq \varepsilon$ for all $1 \leq n \leq N-1$ and $k \geq K$. Consequently,

$$
\sup \left\{\left\|e_{k} x_{n} e_{k}\right\|_{E}: n \in \mathbb{N}\right\} \leq \varepsilon
$$

whenever $k \geq K$. This shows that $\left\{x_{n}\right\}_{n=1}^{\infty}$ has uniformly absolutely continuous norm.

(ii) $\Rightarrow\left(\right.$ i). It is sufficient to show $\left\{x_{n}\right\}_{n=1}^{\infty}$ has a subsequence which converges to zero, since this implies that each subsequence has a subsequence converging to zero. Furthermore, without loss of generality, it may be assumed that $x_{n}^{*}=x_{n}$ for all $n$. It follows from Lemma 6.9 (ii) and Lemma 6.10 (ii) that there exists a $\sigma$-finite projection $p \in P(\mathcal{M})$ such that $x_{n}=p x_{n} p$ for all $n$. Therefore, passing to the reduced von Neumann algebra $p \mathcal{M} p$ and the corresponding strongly symmetrically normed space $p E p$, it may be assumed that $\mathbf{1}$ is $\sigma$-finite. Let $\left\{e_{k}\right\}_{k=1}^{\infty}$ be a sequence in $P(\mathcal{M})$ such that $e_{k} \uparrow \mathbf{1}$ and $\tau\left(e_{k}\right)<\infty$ for all $k$. 
Since $x_{n} \stackrel{\mathcal{T}_{m}}{\rightarrow} 0$, it follows from Lemma 2.3 that, by passing to a subsequence of $\left\{x_{n}\right\}_{n=1}^{\infty}$, it may be assumed that there exists a sequence $\left\{p_{j}\right\}_{j=1}^{\infty}$ in $P(\mathcal{M})$ satisfying $p_{j} \uparrow \mathbf{1}, \tau\left(p_{j}^{\perp}\right) \rightarrow 0$ as $j \rightarrow \infty$ and $\left\|x_{n} p_{j}\right\|_{B(H)} \rightarrow 0$ as $n \rightarrow \infty$ for all $j$.

Observe that

$$
\begin{aligned}
\left\|x_{n}\right\|_{E} & \leq\left\|x_{n} e_{k}\right\|_{E}+\left\|e_{k} x_{n} e_{k}^{\perp}\right\|_{E}+\left\|e_{k}^{\perp} x_{n} e_{k}^{\perp}\right\|_{E} \\
& \leq 2\left\|x_{n} e_{k}\right\|_{E}+\left\|e_{k}^{\perp} x_{n} e_{k}^{\perp}\right\|_{E}
\end{aligned}
$$

since

$$
\left\|e_{k} x_{n} e_{k}^{\perp}\right\|_{E} \leq\left\|e_{k} x_{n}\right\|_{E}=\left\|\left(e_{k} x_{n}\right)^{*}\right\|_{E}=\left\|x_{n} e_{k}\right\|_{E} .
$$

Let $\varepsilon>0$ be given. Since $e_{k}^{\perp} \downarrow 0$, the assumption that $\left\{x_{n}\right\}_{n=1}^{\infty}$ is of uniformly absolutely continuous norm implies that there exists a $k \in \mathbb{N}$ such that

$$
\sup _{n \in \mathbb{N}}\left\|e_{k}^{\perp} x_{n} e_{k}^{\perp}\right\|_{E}<\varepsilon .
$$

Keeping this $k$ fixed, observe that

$$
\begin{aligned}
\left\|x_{n} e_{k}\right\|_{E} & \leq\left\|x_{n} p_{j} e_{k}\right\|_{E}+\left\|p_{j} x_{n} p_{j}^{\perp} e_{k}\right\|_{E}+\left\|p_{j}^{\perp} x_{n} p_{j}^{\perp} e_{k}\right\|_{E} \\
& \leq 2\left\|x_{n} p_{j}\right\|_{B(H)}\left\|e_{k}\right\|_{E}+\left\|p_{j}^{\perp} x_{n} p_{j}^{\perp}\right\|_{E} .
\end{aligned}
$$

Since $p_{j}^{\perp} \downarrow 0$, again the fact that $\left\{x_{n}\right\}_{n=1}^{\infty}$ is of uniformly absolutely continuous norm implies that there exists a $j \in \mathbb{N}$ such that

$$
\sup _{n \in \mathbb{N}}\left\|p_{j}^{\perp} x_{n} p_{j}^{\perp}\right\|_{E}<\varepsilon .
$$

Using the fact that $\left\|x_{n} p_{j}\right\|_{B(H)} \rightarrow 0$ as $n \rightarrow \infty$, choose $N \in \mathbb{N}$ such that $\left\|x_{n} p_{j}\right\|_{B(H)}\left\|e_{k}\right\|_{E}<\varepsilon$ for all $n \geq N$. Combining the above estimates, it follows that $\left\|x_{n}\right\|_{E}<7 \varepsilon$ for all $n \geq N$, and this suffices for the proof of the theorem.

The above proposition has the following consequence.

Proposition 6.12. If $E$ is a strongly symmetrically normed space, then $E^{a n}=E^{o c}$.

Proof. In part (i) of Lemma 6.9, it has already been observed that $E^{o c} \subseteq E^{a n}$. For the proof of the reverse inclusion, it should be observed first that $\left(E^{a n}\right)^{+} \subseteq\left(E^{o c}\right)^{+}$. Indeed, suppose that $0 \leq x \in E^{a n}$ and let $\left\{x_{n}\right\}_{n=1}^{\infty}$ be a sequence in $E$ such that $x \geq x_{n} \downarrow_{n} 0$. By Lemma 6.9 (ii), $x \in S_{0}(\tau)$, and so it follows from [10], Lemma 3.5, that $x_{n} \rightarrow 0$ with respect to the measure topology. Furthermore, $\left\{x_{n}\right\}_{n=1}^{\infty} \subseteq[0, x]$, and so $\left\{x_{n}\right\}_{n=1}^{\infty}$ is of uniformly absolutely continuous norm. Consequently, it follows from Theorem 6.11 that $\left\|x_{n}\right\|_{E} \rightarrow 0$ as $n \rightarrow \infty$. By (6.1), this suffices to show that $x \in E^{o c}$.

Suppose next that $x \in E^{a n}$ and $x^{*}=x$. Setting $p=e^{x}(0, \infty)$, it is clear that $x^{+}=x p=p x p$. If $\left\{p_{n}\right\}_{n=1}^{\infty}$ is a sequence in $P(\mathcal{M})$ such that $p \geq p_{n} \downarrow 0$, then

$$
\left\|p_{n} x^{+} p_{n}\right\|_{E}=\left\|p_{n} p x p p_{n}\right\|_{E}=\left\|p_{n} x p_{n}\right\|_{E} \rightarrow 0, \quad n \rightarrow \infty .
$$

This shows that $x^{+}$is of absolutely continuous norm in the space $p E p$ with respect to the reduced von Neumann algebra $p \mathcal{M} p$. By the first part of the proof, $\left((p E p)^{a n}\right)^{+} \subseteq\left((p E p)^{o c}\right)^{+}$, and hence $x^{+} \in p E p^{o c}$. If $\left\{x_{\alpha}\right\}$ is a net in $E$ satisfying $x^{+} \geq x_{\alpha} \downarrow_{\alpha} 0$, then $x_{\alpha}=p x_{\alpha} p$ for all $\alpha$, and so $x_{\alpha} \downarrow_{\alpha} 0$ in $p E p$. Consequently, $\left\|x_{\alpha}\right\|_{E} \downarrow 0$, which shows that $x^{+} \in E^{o c}$. Similarly, $x^{-}=(-x)^{+} \in E^{o c}$, and hence $x \in E^{o c}$. Since $E^{a n}$ is closed under taking adjoints, this suffices to prove that $E^{a n} \subseteq E^{o c}$. 
The following theorem collects together a number of useful characterizations of elements of absolutely continuous norm.

Theorem 6.13. Suppose that $E \subseteq S(\tau)$ is a strongly symmetrically normed space. If $x \in E$, then the following statements are equivalent:

(i) $x \in E^{a n}$, that is, for all decreasing sequences $\left\{e_{n}\right\}_{n=1}^{\infty}$ in $P(\mathcal{M})$,

$$
e_{n} \downarrow_{n} 0 \Longrightarrow\left\|e_{n} x e_{n}\right\|_{E} \rightarrow_{n} 0
$$

(ii) for all decreasing systems $\left\{e_{\alpha}\right\}$ in $P(\mathcal{M})$,

$$
e_{\alpha} \downarrow_{\alpha} 0 \Longrightarrow\left\|e_{\alpha} x e_{\alpha}\right\|_{E} \rightarrow_{\alpha} 0 \text {; }
$$

(iii) for all decreasing sequences $\left\{e_{n}\right\}_{n=1}^{\infty}$ in $P(\mathcal{M})$,

$$
e_{n} \downarrow_{n} 0 \Longrightarrow\left\|x e_{n}\right\|_{E} \rightarrow_{n} 0 \text {; }
$$

(iv) for all decreasing systems $\left\{e_{\alpha}\right\}$ in $P(\mathcal{M})$,

$$
e_{\alpha} \downarrow_{\alpha} 0 \Longrightarrow\left\|x e_{\alpha}\right\|_{E} \rightarrow_{\alpha} 0
$$

(v) $x \in E^{o c}$, that is, for all decreasing systems $\left\{x_{\alpha}\right\}$ in $E$,

$$
|x| \geq x_{\alpha} \downarrow_{\alpha} 0 \Longrightarrow\left\|x_{\alpha}\right\|_{E} \rightarrow_{\alpha} 0
$$

(vi) for all decreasing sequences $\left\{x_{n}\right\}_{n=1}^{\infty}$ in $E$,

$$
|x| \geq x_{n} \downarrow_{n} 0 \Longrightarrow\left\|x_{n}\right\|_{E} \rightarrow_{n} 0 .
$$

Moreover, if $E$ is a strongly symmetric space, then the above conditions are also equivalent with each of the following two statements:

(vii) for all mutually orthogonal sequences $\left\{e_{n}\right\}_{n=1}^{\infty}$ in $P(\mathcal{M})$ it follows that

$$
\left\|x e_{n}\right\|_{E} \rightarrow_{n} 0
$$

(viii) for all mutually orthogonal sequences $\left\{e_{n}\right\}_{n=1}^{\infty}$ in $P(\mathcal{M})$ it follows that

$$
\left\|e_{n} x e_{n}\right\|_{E} \rightarrow_{n} 0 \text {. }
$$

Proof. The equivalence $(\mathrm{i}) \Leftrightarrow(\mathrm{v})$ has been obtained in Proposition 6.12, and the equivalence $(\mathrm{v}) \Leftrightarrow(\mathrm{vi})$ has been observed (6.1) . The implications (iv) $\Rightarrow($ iii) $\Rightarrow(\mathrm{i})$ and (iv) $\Rightarrow$ (ii) $\Rightarrow($ i) are all evident.

For the proof of implication $(\mathrm{v}) \Rightarrow(\mathrm{iv})$ it suffices to consider positive elements only. Suppose that $x \in E^{+}$satisfies condition $(\mathrm{v})$ and let $\left\{e_{\alpha}\right\}$ be a net in $P(\mathcal{M})$ such that $e_{\alpha} \downarrow_{\alpha} 0$. It follows from Lemma 4.4 that

$$
\left\|x e_{\alpha}\right\|_{E} \leq 4\|x\|_{E}^{1 / 2}\left\|e_{\alpha} x e_{\alpha}\right\|_{E}^{1 / 2}
$$

for all $\alpha$. Furthermore,

$$
\begin{aligned}
\mu\left(e_{\alpha} x e_{\alpha}\right) & =\mu\left(\left(x^{1 / 2} e_{\alpha}\right)^{*}\left(x^{1 / 2} e_{\alpha}\right)\right) \\
& =\mu\left(\left(x^{1 / 2} e_{\alpha}\right)\left(x^{1 / 2} e_{\alpha}\right)^{*}\right)=\mu\left(x^{1 / 2} e_{\alpha} x^{1 / 2}\right),
\end{aligned}
$$

which implies that $x^{1 / 2} e_{\alpha} x^{1 / 2} \in E$ and $\left\|e_{\alpha} x e_{\alpha}\right\|_{E}=\left\|x^{1 / 2} e_{\alpha} x^{1 / 2}\right\|_{E}$ for all $\alpha$. Since

$$
x \geq x^{1 / 2} e_{\alpha} x^{1 / 2} \downarrow_{\alpha} 0,
$$

the assumption on $x$ implies that $\left\|e_{\alpha} x e_{\alpha}\right\|_{E} \rightarrow_{\alpha} 0$. Consequently, it follows from (6.3) that $\left\|x e_{\alpha}\right\|_{E} \rightarrow_{\alpha} 0$. This shows that (v) $\Rightarrow(\mathrm{iv})$, and hence we may conclude that all of the statements (i)-(vi) are equivalent. 
Assume now, in addition, that $E$ is a Banach space. The implication (vii) $\Rightarrow$ (viii) is evident. It will be shown next that (viii) $\Rightarrow(\mathrm{i})$. Suppose first that $x \in E^{+}$ satisfies (viii), and let $\left\{p_{n}\right\}_{n=1}^{\infty} \subseteq P(\mathcal{M})$ satisfy $p_{n} \downarrow_{n} 0$. Defining $y_{n}=x^{1 / 2} p_{n} x^{1 / 2}$, it follows that $y_{n} \downarrow_{n} 0$ and $\left\|e_{n} x e_{n}\right\|_{E}=\left\|y_{n}\right\|_{E}$ for all $n \in \mathbb{N}$. If the sequence $\left\{y_{n}\right\}_{n=1}^{\infty}$ is not Cauchy, then there exist $\varepsilon>0$ and a subsequence $\left\{y_{n_{k}}\right\}_{k=1}^{\infty}$ such that $\left\|y_{n_{k}}-y_{n_{k+1}}\right\|_{E} \geq \varepsilon$ for all $k$. Setting $e_{k}=p_{n_{k}}-p_{n_{k+1}}$, the sequence $\left\{e_{k}\right\}_{k=1}^{\infty} \subseteq$ $P(\mathcal{M})$ is mutually orthogonal and

$$
\left\|e_{k} x e_{k}\right\|_{E}=\left\|x^{1 / 2} e_{k} x^{1 / 2}\right\|_{E}=\left\|y_{n_{k}}-y_{n_{k+1}}\right\|_{E} \geq \varepsilon, \quad k \in \mathbb{N},
$$

which is a contradiction. Consequently, the sequence $\left\{y_{n}\right\}_{n=1}^{\infty}$ is Cauchy and hence convergent in $E$. Since $y_{n} \downarrow_{n} 0$, this implies that $\left\|y_{n}\right\|_{E} \rightarrow 0$ as $n \rightarrow \infty$. This shows that $x \in\left(E^{a n}\right)^{+}$.

Suppose next that $x^{*}=x \in E$ satisfies (viii), and let $p=e^{x}(0, \infty)$. If $\left\{e_{n}\right\}_{n=1}^{\infty} \subseteq$ $P(\mathcal{M})$ is a mutually orthogonal sequence in $P(\mathcal{M})$ such that $e_{n} \leq p$ for all $n$, then $\left\|e_{n} x^{+} e_{n}\right\|_{E}=\left\|e_{n} x p e_{n}\right\|_{E}=\left\|e_{n} x e_{n}\right\|_{E} \rightarrow_{n} 0$. This shows that $x^{+}$satisfies condition (viii) in the space $p E p$. It now follows from the above that $x^{+}$is of absolutely continuous norm in the space $p E p$. As in the last part of the proof of Proposition 6.12, this implies that $x^{+} \in E^{o c}=E^{a n}$. Similarly, $x^{-} \in E^{a n}$, and so $x \in E^{a n}$. Since $x^{*}$ satisfies (viii) whenever $x$ does, this suffices for the proof of implication (viii) $\Rightarrow$ (i).

For the proof that $(\mathrm{i}) \Rightarrow$ (vii), it may be assumed that $x \in\left(E^{a n}\right)^{+}$. Given a mutually orthogonal sequence $\left\{e_{n}\right\}_{n=1}^{\infty} \subseteq P(\mathcal{M})$, define $p_{n} \in P(\mathcal{M})$ by setting $p_{n}=\sum_{k=n}^{\infty} e_{k}$. Since $p_{n} \downarrow 0$ and since we already know that (i) $\Rightarrow$ (iii), it follows from $\left\|x e_{n}\right\|_{E}=\left\|x p_{n} e_{n}\right\|_{E} \leq\left\|x p_{n}\right\|_{E}$ that $\left\|x e_{n}\right\|_{E} \rightarrow 0$ as $n \rightarrow \infty$. The proof is complete.

Since the norm in $E$ is order continuous if and only if $E=E^{o c}$, the following corollary is an immediate consequence of Theorem 6.13.

Corollary 6.14. Let $E \subseteq S(\tau)$ be a strongly symmetric space. The norm in $E$ is order continuous if and only if every $x \in E$ (equivalently, every $x \in E^{+}$) satisfies one (equivalently, all) of the conditions (i)-(viii) in Theorem 6.13.

\section{REFERENCES}

[1] T. Andô, On fundamental properties of a Banach space with a cone, Pacific J. Math. 44 (1962), 1163-1169. MR0150572 (27:568)

[2] A.M. Bikchentaev, The continuity of multiplication for two topologies associated with a semifinite von Neumann algebra, Lobachevskii J. Math. 14 (2004), 17-24. MR2034258 (2005a:46130)

[3] V.I. Chilin and F.A. Sukochev, Weak convergence in non-commutative symmetric spaces, J. Operator Th. 31 (1994), 35-65. MR 1316983 (96e:46085)

[4] J. Diestel, Sequences and Series in Banach Spaces, Springer-Verlag, New York, 1984. MR737004 (85i:46020)

[5] J. Dixmier, Les fonctionelles linéaires sur l'ensemble des opérateurs bornés d'un espace de Hilbert, Annals of Math. 51 (1950), 387-408. MR0033445(11:441e)

[6] J. Dixmier, Von Neumann algebras, North-Holland Mathematical Library, Volume 27, NorthHolland, Amsterdam, 1981. MR641217(83a:46004)

[7] P.G. Dodds, T.K. Dodds, B. de Pagter, Non-commutative Banach function spaces, Math. Z. 201 (1989), 583-597. MR.1004176 (90j:46054)

[8] P.G. Dodds, T.K. Dodds, B. de Pagter, A general Markus inequality, Proc. Centre Math. Anal. Austral. Nat. Univ. 24 (1989), 47-57. MR.1060110(91i:46072) 
[9] P.G. Dodds, T.K. Dodds and B. de Pagter, Weakly compact subsets of symmetric operator spaces, Math. Proc. Camb. Phil. Soc. 110 (1991), 169-182. MR.1104612 (93e:46074)

[10] P.G. Dodds, T.K. Dodds and B. de Pagter, Non-commutative Köthe duality, Trans. Amer. Math. Soc. 339 (1993), 717-750. MR.1113694 (94a:46093)

[11] P.G. Dodds, T.K. Dodds, F.A. Sukochev, O.Ye. Tikhonov, A non-commutative Yosida-Hewitt theorem and convex sets of measurable operators closed locally in measure, Positivity 9 (2005), 457-484. MR2188531 (2006h:46062)

[12] P.G. Dodds and C. Lennard, Normality in trace ideals, J. Operator Th. 16 (1986), 127-145. MR 847335 (87k:47102)

[13] P.G. Dodds and B. de Pagter, Non-commutative Yosida-Hewitt theorems and singular functionals in symmetric spaces of $\tau$-measurable operators, In: Vector Measures, Integration and Related Topics, Proc. of Conf. on "Vector Measures, Integration and Related Topics", Eichstätt, Sept. 2008, G.P. Curbera et. al. (Eds.), Operator Theory: Advances and Applications 201, Birkhäuser Verlag, Basel, 2010, pp. 183-198. MR2743986

[14] Th. Fack and H. Kosaki, Generalized $s$-numbers of $\tau$-measurable operators, Pacific J. Math. 123 (1986), 269-300. MR840845 (87h:46122)

[15] J. Grosberg and M. Krein, Sur la décomposition des fonctionelles en composantes positives, C.R. (Doklady) de l'Acad. Sci. de l'URSS 25 (1939), 723-726. MR0002019 (1:338a)

[16] G.J.O. Jameson, Ordered Linear Spaces, Lecture Notes in Mathematics 141, Springer-Verlag, Berlin, 1970. MR0438077 (55:10996)

[17] R.V. Kadison and J.R. Ringrose, Fundamentals of the theory of operator algebras, Volume I, Academic Press, New York-Orlando, 1983. MR719020 (85j:46099)

[18] R.V. Kadison and J.R. Ringrose, Fundamentals of the theory of operator algebras, Volume II, Academic Press, New York-Orlando, 1983. MR0859186 (88d:46106)

[19] S.G. Krein, Ju.I. Petunin and E.M. Semenov, Interpolation of Linear Operators, Transl. Math. Monographs, Vol. 54, Amer. Math. Soc., Providence, R.I., 1982. MR649411|(84j:46103)

[20] W.A.J. Luxemburg, Banach Function Spaces, Thesis, Delft, 1955. MR0072440 (17:285a)

[21] B. de Pagter, Non-commutative Banach function spaces, in: Positivity, Trends in Mathematics, 197-227 (editors: K. Boulabiar, G. Buskes, A. Triki), Birkhäuser Verlag, Basel, 2007. MR2382219 (2009b:46129)

[22] Narcisse Randrianantoanina, Sequences in non-commutative $L^{p}$-spaces, J. Operator Th. 48 (2002), 255-272. MR 1938797 (2003h:46093)

[23] S. Stratila and L. Zsidó, Lectures on von Neumann Algebras, Editura Abacus Press, 1979. MR.526399 (81j:46089)

[24] M. Takesaki, On the conjugate space of operator algebra, Tôhoku Math. J. 10 (1958), 194-203. MR0100799 (20:7227)

[25] M. Takesaki, On the singularity of a positive linear functional on operator algebra, Proc. Japan. Acad. 35 (1959), 365-366. MR0113153 (22:3991)

[26] M. Takesaki, Theory of Operator Algebras I, Springer-Verlag, Berlin-Heidelberg-New York, 1979. MR548728(81e:46038)

[27] M. Takesaki, Theory of Operator Algebras II, Springer-Verlag, Berlin-Heidelberg-New York, 2003. MR1943006 (2004g:46079)

[28] M. Terp, $L^{p}$ spaces associated with von Neumann algebras, Notes, Copenhagen Univ., 1981.

[29] K. Yosida and E. Hewitt, Finitely additive measures, Trans. Amer. Math Soc. 72 (1952), 46-66. MR0045194 (13:543b)

[30] A.C. Zaanen, Integration, North-Holland, Amsterdam, 1967. MR0222234 (36:5286)

[31] A.C. Zaanen, Riesz Spaces II, North-Holland, Amsterdam-New York-Oxford, 1983. MR704021 (86b:46001)

School of Computer Science, Engineering and Mathematics, Flinders University, GPO Box 2100, Adelaide 5001, Australia

E-mail address: peter@csem.flinders.edu.au

Delft Institute of Applied Mathematics, Faculty Eemcs, Delft University of TechNOlogy, P.O. Box 5031, 2600 GA Delft, The Netherlands

E-mail address: b.depagter@tudelft.nl 